\title{
Uniform growth of groups acting on Cartan-Hadamard spaces
}

Received December 5, 2008 and in revised form July 28, 2010

\begin{abstract}
In this paper we investigate the growth of finitely generated groups. We recall the definition of the algebraic entropy of a group and show that if the group is acting as a discrete subgroup of the isometry group of a Cartan-Hadamard manifold with pinched negative curvature then a Tits alternative is true. More precisely the group is either virtually nilpotent or has a uniform growth bounded below by an explicit constant.
\end{abstract}

\section{Introduction}

In this paper we investigate the growth of finitely generated groups. Given a group $\Gamma$ generated by a finite set $S$, the word length $l_{S}(\gamma)$ of an element $\gamma \in \Gamma$ is the smallest integer $m$ such that there exist elements $\sigma_{1}, \ldots, \sigma_{m}$ in $S \cup S^{-1}$ with $\gamma=\sigma_{1} \ldots \sigma_{m}$. The entropy of $\Gamma$ with respect to the generating set $S$ is defined by

$$
\operatorname{Ent}_{S}(\Gamma)=\lim _{m \rightarrow \infty} \frac{1}{m} \log \sharp\left\{\gamma \in \Gamma \mid l_{S}(\gamma) \leq m\right\} .
$$

If $\operatorname{Ent}_{S}(\Gamma)>0$ for some generating set $S$, it is true for all (finite) generating sets and the group is said to have exponential growth. We now define the algebraic entropy of $\Gamma$,

$$
\operatorname{Ent} \Gamma=\inf _{S}\left\{\operatorname{Ent}_{S}(\Gamma) \mid S \text { a finite generating set of } \Gamma\right\} .
$$

We say that $\Gamma$ has uniform exponential growth if Ent $\Gamma>0$. In [Gro81, remarque 5.12] M. Gromov raised the question whether exponential growth always implies uniform exponential growth. The answer is negative; indeed, in [Wil04] J. S. Wilson gave examples of finitely generated groups of exponential growth but non-uniform exponential growth. Nevertheless, exponential growth implies uniform exponential growth for hyperbolic groups [Kou98], geometrically finite groups of isometries of Cartan-Hadamard manifolds with pinched negative curvature [AN05], solvable groups [Osi03] and linear groups [EMO05], [BrGe08], [Bre08]. For further references see the expository paper [Har02].

We suppose that $(X, g)$ is an $n$-dimensional Cartan-Hadamard manifold of pinched sectional curvature $-a^{2} \leq K_{g} \leq-1$. Our main result is the following theorem.

G. Besson, S. Gallot: UJF-Grenoble 1, CNRS UMR 5582, Institut Fourier, F-38401 Grenoble, France; e-mail: G.Besson@ujf-grenoble.fr, Sylvestre.Gallot@ujf-grenoble.fr

G. Courtois: UPMC-Paris 6, CNRS UMR 7586, Institut de Mathématiques de Jussieu, F-75005 Paris, France; e-mail: Courtois@math.jussieu.fr 
Theorem 1.1. There exists a positive constant $C(n, a)$ such that for any finitely generated discrete group $\Gamma$ of isometries of $(X, g)$, either $\Gamma$ is virtually nilpotent or $\operatorname{Ent}(\Gamma) \geq$ $C(n, a)$.

Remark 1.2. The difficulty is here to show that one can choose the constant $C(n, a)$ not depending on the group $\Gamma$. In the linear setting, E. Breuillard obtained the same kind of uniformity proving the existence of a positive constant $C(n)$ such that for any finitely generated subgroup $\Gamma$ of $G L(n, K), K$ any field, either $\Gamma$ is virtually solvable or $\operatorname{Ent}(\Gamma) \geq C(n)$.

The classical technique is to prove that "not too far" from any finite generating system one can exhibit a free group (on two generators). In this paper we prove this in one of the cases under consideration, using the famous ping-pong lemma; however, in the second case we use a different approach constructing natural Lipschitz maps from the Cayley graph into $X$. This is the new idea which is described in the following.

In a private communication $M$. Kapovich mentioned to us a different proof in the case when $\Gamma$ acts without any elliptic element. One important issue in our proof is that we do not have this restriction: elliptic elements are permitted.

In the forthcoming paper [BCG] we shall use this result to prove a Margulis lemma without curvature; indeed, we shall replace the curvature assumptions by a hypothesis on the growth of the fundamental group.

\section{Preliminaries}

Let $(X, g)$ be an $n$-dimensional Cartan-Hadamard manifold with sectional curvature $-a^{2} \leq K_{g} \leq-1$. Let us recall a few well-known facts about isometries. If $\gamma$ is an isometry of $(X, g)$, the displacement of $\gamma$ is defined by $l(\gamma)=\inf _{x \in X} \rho(x, \gamma x)$, where $\rho$ is the distance associated to the metric $g$ on $X$. We then have (see [Ebe96, p. 31]):

1. The isometry $\gamma$ is called hyperbolic (or axial) if $l(\gamma)>0$, in which case there exists a geodesic $a_{\gamma}$, called the axis of $\gamma$, such that $\rho(x, \gamma x)=l(\gamma)$ for any $x \in a_{\gamma}$.

2. The isometry $\gamma$ is called parabolic if $l(\gamma)=0$ and $l(\gamma)$ is not achieved on $X$, in which case there exists a unique point $\theta$ on the geometric boundary $\partial X$ of $X$ such that $\gamma \theta=\theta$.

3. The isometry $\gamma$ is called elliptic if $l(\gamma)=0$ and $l(\gamma)$ is achieved on $X$, in which case there exists a non-empty convex subset $F_{\gamma}$ of $X$ such that $\gamma x=x$ for any $x \in F_{\gamma}$.

The following result, due to G. Margulis, describes the structure of discrete subgroups of isometries generated by elements with small displacement.

Theorem 2.1 (G. Margulis, [Bur-Zal]). There exists a constant $\mu(n, a)>0$ such that if $\Gamma$ is a discrete subgroup of the isometry group of $(X, g)$ and $x \in X$, then the subgroup $\Gamma_{\mu}(x)$ of $\Gamma$ generated by

$$
S_{\mu}(x)=\{\gamma \in \Gamma \mid \rho(x, \gamma x)<\mu(n, a)\}
$$

is virtually nilpotent. 
Given a set $S=\left\{\sigma_{1}, \ldots, \sigma_{p}\right\}$ of isometries of $(X, g)$, we define the minimal displacement of $S$ by

Definition 2.2. $L(S)=\inf _{x \in X} \max _{i=1, \ldots, p} \rho\left(x, \sigma_{i} x\right)$.

When $\Gamma$ is a finitely generated discrete subgroup of the isometry group of $(X, g)$, the above Theorem 2.1 has the following

Corollary 2.3. There exists a constant $\mu(n, a)>0$ such that if $\Gamma$ is a finitely generated not virtually nilpotent discrete subgroup of the isometry group of $(X, g)$ and $S=\left\{\sigma_{1}, \ldots, \sigma_{p}\right\}$ a finite generating set of $\Gamma$, then

$$
L(S) \geq \mu(n, a) .
$$

In the following lemma we describe the structure of virtually nilpotent discrete groups of isometries of $(X, g)$. Here by discrete we mean that the orbits are discrete sets in $(X, g)$.

Lemma 2.4. Let $\Gamma$ be a discrete virtually nilpotent group of isometries of $(X, g)$.

(a) If $\Gamma$ contains a hyperbolic element $\gamma$, then $\Gamma$ preserves the axis of $\gamma$.

(b) If $\Gamma$ contains a parabolic element $\gamma$ with fixed point $\theta \in \partial X$, then $\Gamma$ fixes $\theta$.

(c) If all elements of $\Gamma$ are elliptic, then $\Gamma$ is finite.

Proof. (a) Let $\gamma \in \Gamma$ be a hyperbolic element and $\theta, \zeta \in \partial X$ the endpoints of the axis $a_{\gamma}$ of $\gamma$. We claim that $\gamma^{\prime}(\{\theta, \zeta\})=\{\theta, \zeta\}$ for any $\gamma^{\prime} \in G$. Indeed, assume for example that $\theta^{\prime}=\gamma^{\prime}(\theta)$ is different from $\theta$ and $\zeta$. The isometry $\gamma^{\prime} \gamma \gamma^{\prime-1}$ is hyperbolic and $\theta^{\prime}$ is one of its fixed points at infinity. By a standard ping-pong argument (see [Gro87, 8.1, p. 211]) we can show that $\Gamma$ contains a free semigroup and hence has exponential growth. On the other hand a virtually nilpotent group has polynomial growth (see [Wol68]), which gives a contradiction.

(b) Let $\gamma \in \Gamma$ be a parabolic element, and $\theta \in \partial X$ its fixed point. If there existed $\gamma^{\prime} \in \Gamma$ such that $\gamma^{\prime} \theta \neq \theta$, then $\gamma$ and $\gamma^{\prime} \gamma \gamma^{\prime-1}$ would be two parabolic elements in $\Gamma$ with distinct fixed points $\theta$ and $\gamma^{\prime} \theta$ respectively. By a ping-pong argument, $\Gamma$ would then contain a free subgroup, which contradicts the fact that $\Gamma$ is virtually nilpotent. Thus $\Gamma$ fixes $\theta \in \partial X$.

(c) Let us now assume that all elements in $\Gamma$ are elliptic. Let $N \subset \Gamma$ be a nilpotent subgroup of $\Gamma$ with finite index. If $N=\{e\}$, then $\Gamma$ is finite. So assume that $N \neq\{e\}$; the center $Z(N)$ of $N$ is then not trivial. For $g_{1} \in Z(N) \backslash\{e\}$ denote by $F_{g_{1}} \subset X$ the set of fixed points of $g_{1}$. Let $x_{1} \in F_{g_{1}}$; as $g_{1}$ and $\exp _{x_{1}}$ commute, we have $F_{g_{1}}=\exp _{x_{1}}\left(E_{1}\right)$, where $E_{1}$ is the eigenspace of $d_{x_{1}} g_{1}$ corresponding to the eigenvalue +1 . This shows that $F_{g_{1}}$ is a totally geodesic submanifold of $X$, furthermore satisfying $\operatorname{dim}\left(F_{g_{1}}\right)<\operatorname{dim}(X)$, since $g_{1} \neq e$. As every $\gamma \in N$ commutes with $g_{1}$, it satisfies $\gamma\left(F_{g_{1}}\right)=F_{g_{1}}$.

Let $N_{1}$ be the subgroup of $\operatorname{Isom}\left(F_{g_{1}}\right)$ obtained by restricting to $F_{g_{1}}$ the elements of $N$; it is clearly nilpotent as the image of a nilpotent group under a morphism. For $\gamma \in N$, the geodesic projection on $F_{g_{1}}$ of any fixed point of $\gamma$ is again a fixed point of $\gamma$; consequently, the elements of $N_{1}$ are elliptic elements of $\operatorname{Isom}\left(F_{g_{1}}\right)$.

If $N_{1}=\{e\}$, then $F_{g_{1}}$ is pointwise fixed by $N$, therefore $N$ is finite (the group is discrete and all elements have a common fixed point). 
If $N_{1} \neq\{e\}$, we may iterate the process. Indeed, suppose that we have constructed the totally geodesic submanifold $F_{g_{i}}$; we then construct $N_{i}$ as the set of restrictions of elements of $N$ to $F_{g_{i}}$, and either $N_{i}=\{e\}$ in which case $N$ is finite, or $N_{i}$ is not trivial, and choosing $g_{i+1} \in Z\left(N_{i}\right) \backslash\{e\}$ we construct the totally geodesic submanifold $F_{g_{i+1}} \subset F_{g_{i}}$ such that $\operatorname{dim}\left(F_{g_{i+1}}\right)<\operatorname{dim}\left(F_{g_{i}}\right)$. This process stops for some $i_{0} \leq n$ and then $N_{i_{0}}=\{e\}$ and $F_{g_{i}}$ is pointwise fixed by $N$ and not empty. Consequently, $N$ is finite.

Lemma 2.5. Let $\Gamma$ be a finitely generated discrete group of isometries of $(X, g)$.

(i) If there exists a point $\theta \in \partial X$ fixed by $\Gamma$, then $\Gamma$ is virtually nilpotent.

(ii) If $\Gamma$ preserves a geodesic in $X$, then $\Gamma$ is virtually cyclic.

Proof. (i) There are two cases:

1) there is a hyperbolic element in $\Gamma$,

2) there is no hyperbolic element, but there is a parabolic element in $\Gamma$ or all elements in $\Gamma$ are elliptic.

1) Let $\gamma$ be a hyperbolic element in $\Gamma$, and $a_{\gamma}$ its axis. One of the endpoints of $a_{\gamma}$ is $\theta$. We claim that for any $\gamma^{\prime} \in \Gamma$, either $\gamma^{\prime}(\{\theta, \zeta\})=\{\theta, \zeta\}$ or $\gamma^{\prime}(\{\theta, \zeta\}) \cap\{\theta, \zeta\}=\varnothing$, where $\zeta$ is the other endpoint of $a_{\gamma}$. Now we finish the proof assuming the claim. Since $\gamma^{\prime}(\theta)=\theta$ by assumption we have $\gamma^{\prime}(\{\theta, \zeta\})=\{\theta, \zeta\}$ and $\gamma^{\prime}(\zeta)=\zeta$. The group $\Gamma$ preserves $a_{\gamma}$. Note that $\Gamma$ does not contain any parabolic element, since such an element would fix $\theta$ and therefore also $\zeta$, which is impossible. The elements in $\Gamma$ are thus either hyperbolic or elliptic.

Now, the projection on $a_{\gamma}$ being distance decreasing, any element $\gamma^{\prime} \in \Gamma$ achieves its displacement $l\left(\gamma^{\prime}\right)$ on the axis $a_{\gamma}$, and $\gamma^{\prime}$ is elliptic (resp. hyperbolic) if and only if $l\left(\gamma^{\prime}\right)=0$ (resp. $l\left(\gamma^{\prime}\right) \neq 0$ ). Moreover, since $\gamma^{\prime}(\theta)=\theta$, any elliptic element fixes pointwise the axis $a_{\gamma}$. The restriction to the axis $a_{\gamma}$ is thus a morphism from $\Gamma$ into the group of translations of the axis, whose kernel is the set of elliptic elements, which fix all points of $a_{\gamma}$. This kernel is then finite and the group $\Gamma$ is virtually cyclic.

Let us now prove the claim. Aiming at a contradiction assume that there exist $\gamma^{\prime} \in \Gamma$ such that $\gamma^{\prime}(\{\theta, \zeta\})=\left\{\theta, \zeta^{\prime}\right\}$ with $\zeta^{\prime} \neq \zeta$. Then $\alpha:=\gamma^{\prime} \gamma\left(\gamma^{\prime}\right)^{-1}$ is a hyperbolic element of $\Gamma$ with axis $a_{\alpha}$ being the geodesic joining $\theta$ and $\zeta^{\prime}$. Assume for example that $a_{\alpha}(-\infty)=\theta$ and fix some point $x \in a_{\alpha}$ so that $\lim _{k \rightarrow \infty} \alpha^{-k}(x)=\theta$. The axes $a_{\alpha}$ and $a_{\gamma}$ are asymptotic at $\theta$, thus $\lim _{k \rightarrow \infty} \rho\left(\alpha^{-k}(x), a_{\gamma}\right)=0$ and therefore there exists $n_{k}$ such that $\gamma^{n_{k}} \alpha^{-k} x$ is a sequence of points which has a subsequence converging to a point $y \in a_{\gamma}$. This contradicts the fact that $\Gamma$ acts properly discontinuously on $X$ and concludes the proof of the claim.

2 ) In this case the elements of $\Gamma$ are either elliptic or parabolic with fixed point $\theta$. In particular, every element of $\Gamma$ preserves globally each horosphere centred at $\theta$. Indeed, this is clear for parabolic elements (see [Ball95, Prop. 3.4, p. 32]). Now, any elliptic element $\gamma^{\prime}$ fixes some point $x \in X$, and hence the whole geodesic $c$ joining $x$ to $\theta$; let $H$ be any horosphere centred at $\theta$ and $y$ be its intersection with $c$; then $\gamma^{\prime}$ maps $H$ onto the horosphere centred at $\gamma^{\prime}(\theta)=\theta$ containing $\gamma^{\prime}(y)=y$. This shows that $\gamma^{\prime}(H)=H$.

Let $S=\left\{\sigma_{1}, \ldots, \sigma_{p}\right\}$ be a generating set of $\Gamma$. By the above discussion we have $\inf _{x \in X} \max _{i \in\{1, \ldots, p\}} \rho(x, \gamma x)=0$. More precisely, for any geodesic $c$ such that 
$c(+\infty)=\theta$, let $H_{t}$ be the horosphere centred at $\theta$ and containing $c(t)$. The orthogonal projection from $H_{t}$ to $H_{t+t^{\prime}}$, for $t^{\prime}>0$, is distance contracting; thus $\rho\left(c(t), \gamma^{\prime}(c(t))\right.$ decreases to zero as $t \rightarrow \infty$, for any $\gamma^{\prime} \in \Gamma$. The group $\Gamma$ is then virtually nilpotent by Corollary 2.3.

Notice that when $\Gamma$ contains only elliptic elements it is finite by Lemma 2.4(c).

(ii) A subgroup of index two of $\Gamma$ fixes each endpoint of the globally preserved geodesic and hence, as before, does not contain any parabolic elements. If it contains a hyperbolic element it is virtually cyclic. If all elements are elliptic they pointwise preserve the geodesic and the group is finite.

For any two isometries $\gamma, \gamma^{\prime}$ acting on $(X, g)$ we define

$$
L\left(\gamma, \gamma^{\prime}\right)=\inf _{x \in X} \max \left\{\rho(x, \gamma x), \rho\left(x, \gamma^{\prime} x\right)\right\}
$$

We now prove the following proposition.

Proposition 2.6. Let $\Gamma$ be a finitely generated discrete subgroup of $\operatorname{Isom}(X, g)$, where $(X, g)$ is a Cartan-Hadamard manifold of sectional curvature $-a^{2} \leq K_{g} \leq-1$. Let $S=\left\{\sigma_{1}, \ldots, \sigma_{p}\right\}$ be a finite generating set of $\Gamma$. If $\Gamma$ is not virtually nilpotent, then either

(i) there exist $\sigma_{i}, \sigma_{j} \in S$ such that the subgroup $\left\langle\sigma_{i}, \sigma_{j}\right\rangle$ generated by these two elements is not virtually nilpotent and hence $L\left(\sigma_{i}, \sigma_{j}\right) \geq \mu(n, a)$; or

(ii) all $\sigma_{i}$ in $S$ are elliptic and for all $\sigma_{i} \neq \sigma_{j} \in S$, either $\left\langle\sigma_{i}, \sigma_{j}\right\rangle$ fixes some point in $X$ and is finite, or it fixes a point $\theta \in \partial X$ and is virtually nilpotent; or

(iii) there exist $\sigma_{i}, \sigma_{j}, \sigma_{k} \in S$ such that $L\left(\sigma_{i} \sigma_{j}, \sigma_{k}\right) \geq \mu(n, a)$ and the group $\left\langle\sigma_{i} \sigma_{j}, \sigma_{k}\right\rangle$ is not virtually nilpotent.

Proof. There are again three cases: (a) there is a hyperbolic element in $S$, say $\sigma_{1}$; (b) there is no hyperbolic element and there is a parabolic element in $S$, say $\sigma_{1}$; (c) all $\sigma_{i}$ 's in $S$ are elliptic.

(a) Assume that $\sigma_{1}$ is hyperbolic. Consider all pairs $\left(\sigma_{1}, \sigma_{i}\right)$ with $i=2, \ldots, p$, and assume that $L\left(\sigma_{1}, \sigma_{i}\right)<\mu(n, a)$ for $i=2, \ldots, p$. The groups $\left\langle\sigma_{1}, \sigma_{i}\right\rangle$ are then virtually nilpotent. By Lemma 2.4(a), every $\sigma_{i}$ preserves the axis $a_{\sigma_{1}}$ of $\sigma_{1}$, hence $\Gamma$ preserves $a_{\sigma_{1}}$ and is virtually nilpotent by Lemma 2.5 , contradicting the assumption. Thus there exists $\sigma_{i} \in S$ such that $L\left(\sigma_{1}, \sigma_{i}\right) \geq \mu(n, a)$ and $\left\langle\sigma_{1}, \sigma_{i}\right\rangle$ is not virtually nilpotent.

(b) Assume that $\sigma_{1}$ is parabolic with fixed point $\theta \in \partial X$. Consider all pairs $\left(\sigma_{1}, \sigma_{i}\right)$, $i=2, \ldots, p$, and assume that $\left\langle\sigma_{1}, \sigma_{i}\right\rangle$ is virtually nilpotent (or $L\left(\sigma_{1}, \sigma_{i}\right)<\mu(n, a)$ for all $i=2, \ldots, p$ ). By Lemma 2.4(b), $\sigma_{i}$ fixes the point $\theta \in \partial X$, therefore $\Gamma$ fixes $\theta$ and is virtually nilpotent, by Lemma 2.5 , a contradiction. Consequently, if $\sigma_{1}$ is parabolic, there exist $\sigma_{i} \neq \sigma_{1}$ such that $L\left(\sigma_{1}, \sigma_{i}\right) \geq \mu(n, a)$.

(c) Assume that all $\sigma_{i}$ 's are elliptic, for $i=2, \ldots, p$, and for all pairs $\left(\sigma_{i}, \sigma_{j}\right)$ the groups $\left\langle\sigma_{i}, \sigma_{j}\right\rangle$ are virtually nilpotent (or $L\left(\sigma_{i}, \sigma_{j}\right)<\mu(n, a)$ ); if one of them is not virtually nilpotent we are in the first case of the alternative. Set $G=\left\langle\sigma_{i}, \sigma_{j}\right\rangle$.

There are again three cases:

1) there is a hyperbolic element in $G$,

2) there is no hyperbolic element and there is a parabolic element in $G$,

3) all elements in $G$ are elliptic. 
In case 1), let $\gamma$ be a hyperbolic element in $G$ with axis $a_{\gamma}$. By Lemma 2.4(a), $G$ preserves $a_{\gamma}$. Since $\sigma_{i}, \sigma_{j}$ are elliptic, they fix points $x_{i}$ and $x_{j}$ (respectively) on $a_{\gamma}$ (recall that the displacements of $\sigma_{i}$ and $\sigma_{j}$ are achieved on $a_{\gamma}$ by the distance decreasing property of the projection onto $a_{\gamma}$ ). If $x_{i}=x_{j}$, then $G$ fixes $x_{i}$ and it is thus finite. Now suppose that $\sigma_{i}$ and $\sigma_{j}$ do not fix the same point on $a_{\gamma}$, that is, $x_{i} \neq x_{j}$ and neither of the restrictions $\tilde{\sigma}_{i}$ and $\tilde{\sigma}_{j}$ of $\sigma_{i}$ and $\sigma_{j}$ to $a_{\gamma}$ is the identity. In that case, $\tilde{\sigma}_{i}$ and $\tilde{\sigma}_{j}$ are both symmetries around points $x_{i}$ and $x_{j}$ of $a_{\gamma}$, and $\sigma_{i} \sigma_{j}$ is a hyperbolic element with axis $a_{\gamma}$. Then consider $\left\langle\sigma_{i} \sigma_{j}, \sigma_{l}\right\rangle$ for $l=1, \ldots, p$. Assume that for all $l=1, \ldots, p$, $L\left(\sigma_{i} \sigma_{j}, \sigma_{l}\right)<\mu(n, a)$. The groups $\left\langle\sigma_{i} \sigma_{j}, \sigma_{l}\right\rangle$ are then virtually nilpotent, and by Lemma 2.4(a), all $\sigma_{l}$ 's preserve $a_{\gamma}$ and hence $\Gamma$ preserves $a_{\gamma}$ and is thus virtually nilpotent, which is a contradiction. Therefore, there exist $\sigma_{k} \in S$ such that $L\left(\sigma_{i} \sigma_{j}, \sigma_{k}\right) \geq \mu(n, a)$ and $\left\langle\sigma_{i} \sigma_{j}, \sigma_{k}\right\rangle$ is not virtually nilpotent.

In case 2), let $\gamma \in G$ be a parabolic element with fixed point $\theta \in \partial X$. By Lemma 2.4(b), $G$ fixes $\theta$.

In case 3), all elements in $G$ are elliptic and by Lemma 2.4(c), $G$ is finite. This ends the proof of the proposition.

\section{Algebraic length and $\eta$-straight isometries}

Let $\Gamma$ be a finitely generated discrete group of isometries of $(X, g)$, and $S=\left\{\sigma_{1}, \ldots, \sigma_{p}\right\}$ be a finite generating set of $\Gamma$.

Let $d_{S}$ denote the distance on the Cayley graph associated to $S$ and recall that $l_{S}$ is the word length on $\Gamma$. Let $x_{0}$ be a point in $X$ and define $L=\max _{i \in\{1, \ldots, p\}} \rho\left(x_{0}, \sigma_{i} x_{0}\right)$.

For any $\gamma \in \Gamma$ it follows from the triangle inequality that

$$
\rho\left(x_{0}, \gamma x_{0}\right) \leq l_{S}(\gamma) L
$$

Let $\eta$ be a positive number such that $0<\eta<L$.

Definition 3.1. An isometry $\gamma$ of $\Gamma$ is said to be $(L, \eta)$-straight if $\rho\left(x_{0}, \gamma x_{0}\right) \geq$ $(L-\eta) l_{S}(\gamma)$.

Remark 3.2. Notice that the above definition depends on the choice of $x_{0}$ and of a generating set $S$.

When $\Gamma$ is a finitely generated discrete group, for any finite generating set $S=$ $\left\{\sigma_{1}, \ldots, \sigma_{p}\right\}$ we recall that the minimal displacement of $S$ is defined (Definition 2.2) by

$$
L(S)=\inf _{x \in X} \max _{i \in\{1, \ldots, p\}} \rho\left(x, \sigma_{i} x\right) .
$$

When $\Gamma$ is not virtually nilpotent, by Theorem 2.1 , for any finite generating set $S, L=$ $L(S) \geq \mu(n, a)>0$, where $\mu(n, a)$ is the Margulis constant. We have 
Lemma 3.3. Let $\Gamma$ be a finitely generated non-virtually nilpotent discrete group of isometries of $(X, g)$. For any finite generating set $S=\left\{\sigma_{1}, \ldots, \sigma_{p}\right\}$ of $\Gamma$, there exists $x_{0} \in X$ such that

$$
L(S)=\inf _{x \in X} \max _{i \in\{1, \ldots, p\}} \rho\left(x, \sigma_{i} x\right)=\max _{i \in\{1, \ldots, p\}} \rho\left(x_{0}, \sigma_{i} x_{0}\right) .
$$

Proof. Assume that the infimum in the definition of $L(S)$ is not achieved in $X$. Then there exists a sequence of points $x_{k} \in X$ which satisfies

$$
\lim _{k \rightarrow \infty} \max _{i \in\{1, \ldots, p\}} \rho\left(x_{k}, \sigma_{i} x_{k}\right)=L(S)
$$

and $x_{k}$ converges to a point, say $\theta$, in $\partial X$. For $k$ large enough and $i \in\{1, \ldots, p\}$, we then have $\rho\left(x_{k}, \sigma_{i} x_{k}\right) \leq L+1$ and hence $\sigma_{i} \theta=\theta$ for all $i$. This shows that $\Gamma$ fixes $\theta$ and is thus virtually nilpotent by Lemma 2.5 , which contradicts the hypothesis.

In the rest of this section, we shall show that if $G$ is a finitely generated discrete group of isometries of $(X, g)$, for any finite generating set $S=\left\{\sigma_{1}, \ldots, \sigma_{p}\right\}$ of $G$ such that each $\sigma_{i}$ has a displacement $l\left(\sigma_{i}\right)$ small compared to $L(S)$, there exist many non- $(L(S), \eta)$-straight elements in $G$ for a constant $\eta$ to be defined.

We need the following geometric lemmas.

Lemma 3.4. Let $\left(x_{1}, x_{2}, x_{3}\right)$ be a geodesic triangle in $(X, g)$, where $(X, g)$ is a CartanHadamard manifold with $K_{g} \leq-1$. Let $x_{2}^{\prime}$ be the point in the segment $\left[x_{1}, x_{3}\right]$ dividing it into two segments of lengths proportional to $L_{1}:=\rho\left(x_{1}, x_{2}\right)$ and $L_{2}:=\rho\left(x_{2}, x_{3}\right)$. Then

$$
\rho\left(x_{2}^{\prime}, x_{2}\right) \leq \operatorname{Argcosh}\left[\exp \left(\alpha\left(\rho\left(x_{1}, x_{2}\right)+\rho\left(x_{2}, x_{3}\right)-\rho\left(x_{1}, x_{3}\right)\right)\right)\right],
$$

where $\alpha=\max \left(L_{1}, L_{2}\right) /\left(L_{1}+L_{2}\right)$.

Proof. We consider a comparison geodesic triangle $\left(y_{1}, y_{2}, y_{3}\right)$ in the Poincaré disk $\left(\mathbb{H}^{2}, d\right)$ of constant curvature -1 such that $d\left(y_{i}, y_{j}\right)=\rho\left(x_{i}, x_{j}\right)$ for all $i, j \in\{1,2,3\}$. Let $y_{2}^{\prime}$ be the point of the segment $\left[y_{1}, y_{3}\right]$ dividing it into two segments of lengths proportional to $L_{1}$ and $L_{2}$. Since $(X, g)$ is a CAT $(-1)$ space we have

$$
\rho\left(x_{2}, x_{2}^{\prime}\right) \leq d\left(y_{2}, y_{2}^{\prime}\right) .
$$

One of the two triangles $\left(y_{1}, y_{2}^{\prime}, y_{2}\right),\left(y_{3}, y_{2}^{\prime}, y_{2}\right)$ has angle at $y_{2}^{\prime}$ greater than or equal to $\pi / 2$, therefore from the hyperbolic trigonometry formulae we get the existence of $i \in\{1,2\}$ such that

$$
\cosh L_{i} \geq \cosh \left[d\left(y_{2}, y_{2}^{\prime}\right)\right] \cosh \left[\frac{L_{i}}{L_{1}+L_{2}} d\left(y_{1}, y_{3}\right)\right]
$$

Set $\Delta=\rho\left(x_{1}, x_{2}\right)+\rho\left(x_{2}, x_{3}\right)-\rho\left(x_{1}, x_{3}\right)=L_{1}+L_{2}-\rho\left(x_{1}, x_{3}\right)$. We have

$$
\frac{L_{i}}{L_{1}+L_{2}} d\left(y_{1}, y_{3}\right) \geq L_{i}-\alpha \Delta,
$$


where $\alpha=\max \left(L_{1}, L_{2}\right) /\left(L_{1}+L_{2}\right)$. Therefore from (4) and (5) we get

$$
\cosh \left[\rho\left(x_{2}^{\prime}, x_{2}\right)\right] \leq \frac{\cosh L_{i}}{\cosh \left(L_{i}-\alpha \Delta\right)}
$$

hence

$$
\cosh \left[\rho\left(x_{2}^{\prime}, x_{2}\right)\right] \leq e^{\alpha \Delta}
$$

Lemma 3.5. Let $(X, g)$ be a Cartan-Hadamard manifold with sectional curvature $K_{g} \leq-1$. Let $\delta, L$ be any positive numbers such that $L>\operatorname{Argcosh} e^{\delta}$. Then, for any isometry $\gamma$ of $(X, g)$ whose displacement satisfies $l(\gamma) \leq \delta$, and for any $x_{0} \in X$ such that $\rho\left(x_{0}, \gamma x_{0}\right) \geq L$, we have

$$
\rho\left(x_{0}, \gamma^{2} x_{0}\right) \leq 2 \rho\left(x_{0}, \gamma x_{0}\right)-\left(1-\frac{e^{\delta}}{\cosh L}\right)^{2} .
$$

Proof. Set $\Delta=2 \rho\left(x_{0}, \gamma x_{0}\right)-\rho\left(x_{0}, \gamma^{2} x_{0}\right)$. We want to prove that $\Delta \geq\left(1-e^{\delta} / \cosh L\right)^{2}$. By assumption there is a point $y \in X$ such that $\rho(y, \gamma y) \leq \delta$. Write $L_{1}:=\rho\left(x_{0}, \gamma y\right)$, $L_{2}:=\rho\left(\gamma^{2} x_{0}, \gamma y\right)$ and $L^{\prime}:=\rho\left(x_{0}, y\right)$. By the triangle inequality we have, for $i=1,2$,

$$
L^{\prime}-\delta \leq L_{i} \leq L^{\prime}+\delta \text {. }
$$

Let us associate to the triangle $\left(x_{0}, \gamma y, \gamma^{2} x_{0}\right)$ the comparison triangle $\left(z_{1}, z_{2}, z_{3}\right)$ in the hyperbolic plane $\left(\mathbb{H}^{2}, d\right)$ such that $d\left(z_{1}, z_{2}\right)=L_{1}, d\left(z_{2}, z_{3}\right)=L_{2}$ and $d\left(z_{1}, z_{3}\right)=$ $\rho\left(x_{0}, \gamma^{2} x_{0}\right)$. Let $x$ (resp. $z$ ) be the middle point of the segment $\left(x_{0}, \gamma^{2} x_{0}\right)$ (resp. $\left(z_{1}, z_{3}\right)$ ). One of the two triangles $\left(z_{2}, z, z_{1}\right)$ or $\left(z_{2}, z, z_{3}\right)$, say the former, has angle at $z$ greater than or equal to $\pi / 2$. Then the hyperbolic trigonometric formulas give

$$
\cosh L_{1} \geq \cosh \left[d\left(z_{2}, z\right)\right] \cosh \left[\frac{1}{2} d\left(z_{1}, z_{3}\right)\right],
$$

therefore from (9) we get

$$
\cosh \left(L^{\prime}+\delta\right) \geq \cosh \left[d\left(z_{2}, z\right)\right] \cosh \left[\frac{1}{2} d\left(z_{1}, z_{3}\right)\right],
$$

and since $(X, g)$ is a CAT $(-1)$ space we also have $\rho(x, \gamma y) \leq d\left(z, z_{2}\right)$. We thus obtain

$$
\cosh \left(L^{\prime}+\delta\right) \geq \cosh [\rho(x, \gamma y)] \cosh \left[\frac{1}{2} \rho\left(x_{0}, \gamma^{2} x_{0}\right)\right] \text {. }
$$

Write $L_{0}=\rho\left(x_{0}, \gamma x_{0}\right)$. By the triangle inequality

$$
\rho(x, \gamma y) \geq\left|\rho\left(\gamma y, \gamma x_{0}\right)-\rho\left(\gamma x_{0}, x\right)\right|,
$$

therefore, since $\rho\left(\gamma y, \gamma x_{0}\right)=\rho\left(y, x_{0}\right)=L^{\prime}$ and $\frac{1}{2} \rho\left(\gamma^{2} x_{0}, x_{0}\right)=L_{0}-\Delta / 2$, from (10) we get

$$
\cosh \left(L^{\prime}+\delta\right) \geq \cosh \left(L^{\prime}-\rho\left(\gamma x_{0}, x\right)\right) \cosh \left(L_{0}-\Delta / 2\right) .
$$


From (11),

$$
\begin{aligned}
& (\cosh \delta+\sinh \delta) \cosh L^{\prime} \\
& \geq\left(\cosh \left[\rho\left(\gamma x_{0}, x\right)\right]-\sinh \left[\rho\left(\gamma x_{0}, x\right)\right]\right)\left(\cosh L^{\prime}\right) \cosh \left(L_{0}-\Delta / 2\right),
\end{aligned}
$$

hence

$$
e^{\delta} \geq\left(\cosh \left[\rho\left(\gamma x_{0}, x\right)\right]-\sinh \left[\rho\left(\gamma x_{0}, x\right)\right]\right) \cosh \left(L_{0}-\Delta / 2\right) .
$$

Now applying the inequality (7) from the proof of Lemma 3.4 we have

$$
\cosh \left[\rho\left(\gamma x_{0}, x\right)\right] \leq \frac{\cosh L_{0}}{\cosh \left(L_{0}-\Delta / 2\right)},
$$

and since $\cosh r-\sinh r=e^{-r}$ is a decreasing function of $r$, from (12) we get

$$
e^{\delta} \geq \cosh L_{0}-\left(\cosh ^{2} L_{0}-\cosh ^{2}\left(L_{0}-\Delta / 2\right)\right)^{1 / 2} .
$$

But we can check that $\cosh ^{2} L_{0}-\cosh ^{2}\left(L_{0}-\Delta / 2\right) \leq \Delta \cosh ^{2} L_{0}$, so (13) yields

$$
e^{\delta} \geq \cosh \left(L_{0}\right)\left(1-\Delta^{1 / 2}\right)
$$

and therefore

$$
\Delta \geq\left(1-\frac{e^{\delta}}{\cosh L_{0}}\right)^{2}
$$

when $e^{\delta}<\cosh L_{0}$, which follows from $e^{\delta}<\cosh L$ and $L_{0} \geq L$.

Lemma 3.6. Let $(X, g)$ be a Cartan-Hadamard manifold with sectional curvature $K_{g} \leq-1$. Consider four points $y_{0}, y_{1}, y_{2}, y_{3}$ such that

$$
\begin{aligned}
& \rho\left(y_{0}, y_{1}\right)+\rho\left(y_{1}, y_{2}\right)-\rho\left(y_{0}, y_{2}\right) \leq \eta_{1}, \\
& \rho\left(y_{1}, y_{2}\right)+\rho\left(y_{2}, y_{3}\right)-\rho\left(y_{1}, y_{3}\right) \leq \eta_{2} .
\end{aligned}
$$

Then

$$
\rho\left(y_{0}, y_{1}\right)+\rho\left(y_{1}, y_{2}\right)+\rho\left(y_{2}, y_{3}\right)-\rho\left(y_{0}, y_{3}\right) \leq\left(1+\frac{\rho\left(y_{2}, y_{3}\right)}{\rho\left(y_{1}, y_{2}\right)}\right)\left(\eta_{1}+\operatorname{Argcosh} e^{\eta_{2}}\right) .
$$

Proof. For $i=1,2,3$ write $L_{i}=\rho\left(y_{i-1}, y_{i}\right)$. Let $y_{2}^{\prime}$ be the point on the segment $\left(y_{1}, y_{3}\right)$ dividing it into two segments of lengths proportional to $L_{2}$ and $L_{3}$. By Lemma 3.4,

$$
\rho\left(y_{2}, y_{2}^{\prime}\right) \leq \operatorname{Argcosh} e^{\eta_{2}} .
$$

Since $\rho\left(y_{0}, y_{1}\right)+\rho\left(y_{1}, y_{2}\right)-\rho\left(y_{0}, y_{2}\right) \leq \eta_{1}$ by assumption, from (14) and the triangle inequality we get

$\rho\left(y_{0}, y_{2}^{\prime}\right) \geq \rho\left(y_{0}, y_{2}\right)-\rho\left(y_{2}, y_{2}^{\prime}\right) \geq \rho\left(y_{0}, y_{1}\right)+\rho\left(y_{1}, y_{2}\right)-\left(\eta_{1}+\operatorname{Argcosh} e^{\eta_{2}}\right)$.

On the other hand by convexity of the distance function on $(X, g)$ we get

$$
\rho\left(y_{0}, y_{2}^{\prime}\right) \leq \frac{L_{3}}{L_{2}+L_{3}} \rho\left(y_{0}, y_{1}\right)+\frac{L_{2}}{L_{2}+L_{3}} \rho\left(y_{0}, y_{3}\right) .
$$


The inequalities (15) and (16) give

$$
\rho\left(y_{0}, y_{3}\right) \geq \rho\left(y_{0}, y_{1}\right)+L_{2}+L_{3}-\frac{L_{2}+L_{3}}{L_{2}} \eta_{1}+\operatorname{Argcosh} e^{\eta_{2}},
$$

and the lemma follows.

Lemma 3.7. Let $L$ and $\eta$ be positive numbers such that

$$
\eta<\min \left(L / 4, \log \left[\frac{1}{2}\left(\cosh (L / 2)+\frac{1}{\cosh (L / 2)}\right)\right]\right) .
$$

Let $(X, g)$ be a Cartan-Hadamard manifold with sectional curvature $K_{g} \leq-1$. Consider two elliptic isometries $\gamma_{1}, \gamma_{2}$ of $(X, g)$ with a common fixed point $y \in X \cup \partial X$. If $L-\eta \leq$ $\rho\left(x_{0}, \gamma_{1} x_{0}\right) \leq L$ and $L-\eta \leq \rho\left(x_{0}, \gamma_{2} x_{0}\right) \leq L$, then

$$
\rho\left(x_{0}, \gamma_{1} \gamma_{2} x_{0}\right)<2(L-\eta) .
$$

Proof. We first claim that in both cases, $y \in X$ and $y \in \partial X$, there exists some sequence $\left(u_{k}\right)_{k \in \mathbb{N}}$ of points in $X$ converging to $y$ such that $\rho\left(u_{k}, \gamma_{1} \gamma_{2} x_{0}\right)=\rho\left(u_{k}, x_{0}\right)=l_{k}$, and that the quantity $\epsilon_{k}=\left|\rho\left(u_{k}, \gamma_{1} x_{0}\right)-l_{k}\right|$ goes to zero as $k \rightarrow \infty$; in fact, when $\gamma_{1}$ and $\gamma_{2}$ fix some point $y \in X$ we may choose $u_{k}=y$ for every $k$. If $\gamma_{1}$ and $\gamma_{2}$ fix $y \in \partial X$, they also preserve each horosphere centred at $y$ (see the proof of Lemma 2.5(ii)), and thus $x_{0}$, $\gamma_{1} x_{0}$ and $\gamma_{1} \gamma_{2} x_{0}$ lie on the same horosphere centred at $y$. Approximating this horosphere by a sequence $\left(S_{k}\right)_{k \in \mathbb{N}}$ of spheres passing through $x_{0}$ and $\gamma_{1} \gamma_{2} x_{0}$ and denoting by $u_{k}$ the centre of $S_{k}$, we see that $\rho\left(u_{k}, \gamma_{1} x_{0}\right)-\rho\left(O, u_{k}\right)$ and $\rho\left(u_{k}, x_{0}\right)-\rho\left(O, u_{k}\right)$ simultaneously go to $B\left(\gamma_{1} x_{0}, y\right)=B\left(x_{0}, y\right)$ (where $O$ is some fixed origin in $X$, and $B$ the Busemann function normalised at $O)$. This proves the claim.

Consider the triangle $\left(u_{k}, v, w\right)=\left(u_{k}, x_{0}, \gamma_{1} \gamma_{2} x_{0}\right)$ and the point $z$ of the geodesic segment $[v, w]$ which divides it into two segments of lengths proportional to $L_{1}:=$ $\rho\left(v, \gamma_{1} x_{0}\right)$ and $L_{2}:=\rho\left(w, \gamma_{1} x_{0}\right)$. Recall that by assumption $L-\eta \leq L_{i} \leq L$.

We consider the comparison triangle $\left(\bar{u}_{k}, \bar{v}, \bar{w}\right)$ on the two-dimensional hyperbolic space $\mathbb{H}^{2}$ such that $d\left(\bar{u}_{k}, \bar{v}\right)=\rho\left(u_{k}, v\right)=l_{k}=\rho\left(u_{k}, w\right)=d\left(\bar{u}_{k}, \bar{w}\right)$ and $d(\bar{v}, \bar{w})=$ $\rho(v, w)$, where $d$ is the hyperbolic distance on $\mathbb{H}^{2}$. Let $\bar{z}$ be the point of the segment $[\bar{v}, \bar{w}]$ dividing it into two segments of lengths proportional to $L_{1}$ and $L_{2}$. Write $L_{1}^{\prime}=\rho(v, z)$ and $L_{2}^{\prime}=\rho(w, z)$. We now consider the triangle $\left(\bar{u}_{k}, \bar{v}, \bar{z}\right)$ or $\left(\bar{u}_{k}, \bar{w}, \bar{z}\right)$ which has angle at $\bar{z}$ larger than or equal to $\pi / 2$. The hyperbolic trigonometry formulas then show that

$$
\cosh l_{k} \geq \cosh L_{1}^{\prime} \cosh \left[d\left(\bar{u}_{k}, \bar{z}\right)\right] .
$$

Since $(X, g)$ is a CAT $(-1)$ space we get

$$
\rho\left(u_{k}, z\right) \leq d\left(\bar{u}_{k}, \bar{z}\right)
$$

and thus

$$
\cosh \left[\rho\left(u_{k}, z\right)\right] \leq \frac{\cosh l_{k}}{\cosh L_{1}^{\prime}}
$$


On the other hand, the triangle inequality implies that $\rho\left(u_{k}, z\right) \geq l_{k}-\epsilon_{k}-\rho\left(\gamma_{1} x_{0}, z\right)$ and thus

$$
\cosh \left[\rho\left(u_{k}, z\right)\right] \geq e^{-\left(\rho\left(\gamma_{1} x_{0}, z\right)+\epsilon_{k}\right)} \cosh l_{k} .
$$

Plugging this into (17) and letting $\epsilon_{k} \rightarrow 0$, we get

$$
e^{\rho\left(\gamma_{1} x_{0}, z\right)} \geq \cosh L_{1}^{\prime}
$$

On the other hand, by Lemma 3.4, we have

$\cosh \left[\rho\left(\gamma_{1} x_{0}, z\right)\right] \leq \exp \left(\max \left\{\rho\left(v, \gamma_{1} x_{0}\right), \rho\left(w, \gamma_{1} x_{0}\right)\right\}\left(1-\frac{\rho(v, w)}{\rho\left(v, \gamma_{1} x_{0}\right)+\rho\left(w, \gamma_{1} x_{0}\right)}\right)\right)$

and hence

$$
\cosh \left[\rho\left(\gamma_{1} x_{0}, z\right)\right] \leq e^{L-\rho(v, w) / 2} .
$$

Now assume, for contradiction, that

$$
\rho(v, w)=\rho\left(x_{0}, \gamma_{1} \gamma_{2} x_{0}\right)>2(L-\eta) .
$$

Plugging this in (18) and (19) we obtain, using the fact that $x \mapsto x+1 / x$ is an increasing function for $x>1$,

$$
\cosh L_{1}^{\prime}+\frac{1}{\cosh L_{1}^{\prime}} \leq 2 \cosh \left[\rho\left(\gamma_{1} x_{0}, z\right)\right] \leq 2 e^{\eta}
$$

Now since $L_{1}^{\prime} / L_{2}^{\prime}=L_{1} / L_{2}$, we also obtain

$$
L_{1}^{\prime}=\left(L_{1}^{\prime}+L_{2}^{\prime}\right) \frac{L_{1}}{L_{1}+L_{2}} \geq \frac{2(L-\eta)(L-\eta)}{2 L} \geq L-2 \eta
$$

which gives, by (20),

$$
\cosh (L-2 \eta)+\frac{1}{\cosh (L-2 \eta)} \leq 2 \cosh \left[\rho\left(\gamma_{1} x_{0}, z\right)\right] \leq 2 e^{\eta}
$$

We then get a contradiction when

$$
\eta<\min \left(L / 4, \log \left[\frac{1}{2}\left(\cosh (L / 2)+\frac{1}{\cosh (L / 2)}\right)\right]\right) .
$$

Let $\Gamma$ be a finitely generated discrete group of isometries of $(X, g)$ and $S=\left\{\sigma_{1}, \ldots, \sigma_{p}\right\}$ be a finite generating set. Assume that $\Gamma$ is not virtually nilpotent and recall that $L(S)=$ $\inf _{x \in X} \max _{i \in\{1, \ldots, p\}} \rho\left(x, \sigma_{i} x\right)$. By Lemma 3.3 we have $L(S)=\max _{i \in\{1, \ldots, p\}} \rho\left(x_{0}, \sigma_{i} x_{0}\right)$ for some $x_{0} \in X$, and by Corollary $2.3, L(S) \geq \mu(n, a)>0$. Recall that for $0 \leq \eta \leq L$, an element $\gamma \in \Gamma$ is said to be $(L, \eta)$-straight if

$$
\rho\left(x_{0}, \gamma x_{0}\right)>(L-\eta) l_{S}(\gamma) .
$$

In the following two propositions we give conditions under which there are many non$(L, \eta)$-straight elements in $\Gamma$. 
Proposition 3.8. Let $(X, g)$ be a Cartan-Hadamard manifold whose sectional curvature satisfies $-a^{2} \leq K_{g} \leq-1$, and $\Gamma$ a discrete non-virtually nilpotent group of isometries of $(X, g)$ generated by $S=\left\{\sigma_{1}, \ldots, \sigma_{p}\right\}$. Assume that all $\sigma_{i}$ 's are elliptic and that for all $\sigma_{i} \neq \sigma_{j} \in S$, the group $\left\langle\sigma_{i}, \sigma_{j}\right\rangle$ fixes a point $y \in X$ or $\theta \in \partial X$. Let $\eta$ be a positive number such that

$$
\eta<\min \left(L / 4, \frac{1}{2}\left(1-\frac{1}{\cosh L}\right)^{2}, \log \left[\frac{1}{2}\left(\cosh (L / 2)+\frac{1}{\cosh (L / 2)}\right)\right]\right),
$$

where $L=L(S)=\max _{i \in\{1, \ldots, p\}} \rho\left(x_{0}, \sigma_{i} x_{0}\right)$. Then any $\gamma \in \Gamma$ with $l_{S}(\gamma)=2$, i.e $\gamma=\sigma_{i}^{ \pm 2}$ or $\gamma=\sigma_{i}^{ \pm 1} \sigma_{j}^{ \pm 1}$, is not $(L, \eta / 2)$-straight, that is, $\rho\left(x_{0}, \gamma x_{0}\right) \leq 2(L-\eta / 2)$.

Proof. Consider the case where $\gamma=\sigma_{i}^{2}$. If $\sigma_{i}$ is not $(L, \eta)$-straight, we have, by the triangle inequality, $\rho\left(x_{0}, \sigma_{i}^{2} x_{0}\right) \leq 2(L-\eta)$. If $\sigma_{i}$ is $(L, \eta)$-straight, by Lemma 3.5 we have, with $\delta=0$,

$$
\rho\left(x_{0}, \sigma_{i}^{2} x_{0}\right) \leq 2 L-\left(1-\frac{1}{\cosh L}\right)^{2} \leq 2(L-\eta) .
$$

Now consider the case where $\gamma=\sigma_{i} \sigma_{j}$ for $i \neq j$. If $\sigma_{i}$ or $\sigma_{j}$ is not $(L, \eta)$-straight, we have, by the triangle inequality,

$$
\rho\left(x_{0}, \sigma_{i} \sigma_{j} x_{0}\right) \leq \rho\left(x_{0}, \sigma_{i} x_{0}\right)+\rho\left(x_{0}, \sigma_{j} x_{0}\right) \leq L+(L-\eta),
$$

therefore, $\rho\left(x_{0}, \sigma_{i} \sigma_{j} x_{0}\right) \leq 2(L-\eta / 2)$.

If $\sigma_{i}$ and $\sigma_{j}$ are $(L, \eta)$-straight, Lemma 3.7 implies $\rho\left(x_{0}, \sigma_{i} \sigma_{j} x_{0}\right) \leq 2(L-\eta)$.

In the next proposition we will assume that all elements $\gamma \in \Gamma$ whose algebraic length is less than or equal to 4 have a displacement smaller than $\delta$ where

$$
\delta=\log \cosh (L / 4)
$$

and we set

$$
\eta=10^{-3}\left(1-\frac{\cosh (L / 4)}{\cosh (L / 2)}\right)^{4}
$$

We will find in that case many non- $(L, \eta)$-straight elements.

Proposition 3.9. Let $(X, g)$ be a Cartan-Hadamard manifold whose sectional curvature satisfies $-a^{2} \leq K_{g} \leq-1$, and $G$ a discrete non-virtually nilpotent group of isometries of $(X, g)$ generated by a set $\Sigma=\left\{\sigma_{1}, \sigma_{2}\right\}$ of two isometries. Let $L=$ $\inf _{x \in X} \max \left\{\rho\left(x, \sigma_{1} x\right), \rho\left(x, \sigma_{2} x\right)\right\}$. Let $\eta$ and $\delta$ be the numbers defined in (23) and (22). Assume that $l\left(\gamma^{\prime}\right)<\delta$ for all $\gamma^{\prime} \in G$ such that $l_{\Sigma}\left(\gamma^{\prime}\right) \leq 4$. Then no $\gamma \in G$ such that $l_{\Sigma}(\gamma)=6$ is $(L, \eta)$-straight.

Recall that $x_{0}$ satisfies $L=\max \left\{\rho\left(x_{0}, \sigma_{1} x_{0}\right), \rho\left(x_{0}, \sigma_{2} x_{0}\right)\right\}$. We will need the following lemmas. 
Lemma 3.10. Let $\gamma=a \gamma^{\prime} b \in G$ be such that $l_{\Sigma}(\gamma)=l_{\Sigma}(a)+l_{\Sigma}\left(\gamma^{\prime}\right)+l_{\Sigma}(b)$. If $\gamma$ is $(L, \eta)$-straight, then $\gamma^{\prime}$ is $(L, C \eta)$-straight where $C=l_{\Sigma}(\gamma) / l_{\Sigma}\left(\gamma^{\prime}\right)$.

Proof. Note that by the definition of $L=L(\Sigma)$, for any $\gamma \in G$ we have

$$
\rho\left(x_{0}, \gamma x_{0}\right) \leq L l_{\Sigma}(\gamma) .
$$

By the triangle inequality

$$
\rho\left(x_{0}, \gamma x_{0}\right) \leq \rho\left(x_{0}, a x_{0}\right)+\rho\left(x_{0}, \gamma^{\prime} x_{0}\right)+\rho\left(x_{0}, b x_{0}\right),
$$

hence by the assumption on $\gamma$ we get

$$
(L-\eta) l_{\Sigma}\left(a \gamma^{\prime} b\right) \leq L\left(l_{\Sigma}(a)+l_{\Sigma}(b)\right)+\rho\left(x_{0}, \gamma^{\prime} x_{0}\right),
$$

and therefore

$$
\rho\left(x_{0}, \gamma^{\prime} x_{0}\right) \geq L l_{\Sigma}\left(\gamma^{\prime}\right)-\eta l_{\Sigma}(\gamma) \geq(L-C \eta) l_{\Sigma}\left(\gamma^{\prime}\right) .
$$

Lemma 3.11. Let $\alpha, \beta$ be elements of $G$ different from the identity and such that $l_{\Sigma}(\alpha) \leq 2$ and $l_{\Sigma}(\beta) \leq 2$. Under the assumptions of Proposition 3.9, if $\gamma$ is $(L, \eta)$ straight with $l_{\Sigma}(\gamma)=6$, then no reduced word representing $\gamma$ contains (i) $\alpha^{2}$ or (ii) $\alpha \beta \alpha$ (here $\alpha \beta \alpha$ is supposed to be reduced).

Assuming Lemma 3.11, the proof of Proposition 3.9 can be finished as follows:

Proof of Proposition 3.9. Let $\gamma \in G$ have length $l_{\Sigma}(\gamma)=6$. Write $\gamma$ as a reduced word in the generators of $\Sigma, \gamma=\sigma_{i_{1}}^{p_{1}} \ldots \sigma_{i_{k}}^{p_{k}}$, where $\sigma_{i_{j}}=\sigma_{1}$ or $\sigma_{i_{j}}=\sigma_{2}, p_{j} \in$ $\mathbb{Z}^{*}, i_{j} \neq i_{j+1}$ and $i_{j}=i_{j+2}$. For a contradiction assume that $\gamma$ is $\eta$-straight. Then, by Lemma 3.11(i), all $p_{j}$ are equal to +1 or -1 and in particular $k=6$. Therefore $\gamma=\sigma_{i_{1}}^{p_{1}} \sigma_{i_{2}}^{p_{2}} \sigma_{i_{3}}^{p_{3}} \sigma_{i_{4}}^{p_{4}} \sigma_{i_{5}}^{p_{5}} \sigma_{i_{6}}^{p_{6}}$. By Lemma 3.11(ii) we also have $p_{j+2} \neq p_{j}$, hence $p_{j+2}=$ $-p_{j}$ so $\gamma=\sigma_{i_{1}}^{p_{1}} \sigma_{i_{2}}^{p_{2}} \sigma_{i_{1}}^{-p_{1}} \sigma_{i_{2}}^{-p_{2}} \sigma_{i_{1}}^{p_{1}} \sigma_{i_{2}}^{p_{2}}$, which is impossible by Lemma 3.11(ii) with $\alpha=\sigma_{i_{1}}^{p_{1}} \sigma_{i_{2}}^{p_{2}}$ and $\beta=\sigma_{i_{1}}^{-p_{1}} \sigma_{i_{2}}^{-p_{2}}$.

Let us now prove Lemma 3.11:

Proof of Lemma 3.11. We first claim that if $L, \eta$ and $\delta$ are as in Proposition 3.9 then

$$
\eta \leq \frac{L}{4000}
$$

and

$$
12 \eta+\operatorname{Argcosh} e^{12 \eta} \leq \frac{1}{4}\left(1-\frac{e^{\delta}}{\cosh (L / 2)}\right) .
$$

Indeed, by the definition of $\eta$ (cf. (23)), we have

$$
1000 \eta=\left(1-\frac{\cosh (L / 4)}{\cosh (L / 2)}\right)^{4}
$$


therefore

$$
1000 \eta<\frac{\cosh (L / 2)-\cosh (L / 4)}{\cosh (L / 2)}
$$

and

$$
1000 \eta<\frac{\sinh (L / 2) \cdot L / 4}{\cosh (L / 2)}<\frac{L}{4},
$$

which proves (24). On the other hand, if $x \in] 0,1\left[\right.$, then $e^{x} \leq 1+2 x \leq \cosh (2 \sqrt{x})$. Choosing $x=12 \eta$ we deduce, using (23) and $\eta<1 / 1000$, that

$$
12 \eta+\operatorname{Argcosh} e^{12 \eta} \leq 12 \eta+2 \sqrt{12 \eta}<\frac{1}{4} \sqrt{1000 \eta},
$$

therefore we get

$$
12 \eta+\operatorname{Argcosh} e^{12 \eta} \leq \frac{1}{4}\left(1-\frac{\cosh (L / 4)}{\cosh (L / 2)}\right)^{2} \leq \frac{1}{4}\left(1-\frac{e^{\delta}}{\cosh (L / 2)}\right),
$$

proving (25).

We now prove (i) of Lemma 3.11. Assume that $\gamma=a \alpha^{2} b$ is $(L, \eta)$-straight and $l_{\Sigma}(\gamma)=6$. Then, by Lemma 3.10, $\alpha$ is $(L, 6 \eta)$-straight and $\alpha^{2}$ is $(L, 3 \eta)$-straight. Hence, by (24),

$$
\rho\left(x_{0}, \alpha x_{0}\right) \geq(L-6 \eta) l_{\Sigma}(\alpha)>L / 2 .
$$

On the other hand since $l(\alpha) \leq \delta$ and $\operatorname{Argcosh} e^{\delta}=L / 4<L / 2$, we can apply Lemma 3.5 to $\alpha$ replacing $L$ by $L / 2$ and get

$$
(L-3 \eta) l_{\Sigma}\left(\alpha^{2}\right) \leq \rho\left(x_{0}, \alpha^{2} x_{0}\right) \leq 2 \rho\left(x_{0}, \alpha x_{0}\right)-\left(1-\frac{e^{\delta}}{\cosh (L / 2)}\right)^{2} .
$$

Hence

$$
\rho\left(x_{0}, \alpha^{2} x_{0}\right) \leq 2 L l_{\Sigma}(\alpha)-\rho\left(x_{0}, \alpha^{2} x_{0}\right) \leq l_{\Sigma}\left(\alpha^{2}\right)\left(L-\frac{1}{4}\left(1-\frac{e^{\delta}}{\cosh (L / 2)}\right)^{2}\right),
$$

where we used that $\alpha^{2}$ is reduced and $l_{\Sigma}\left(\alpha^{2}\right) \leq 4$. Then by the choice of $\eta$ (cf. (23)),

$$
\rho\left(x_{0}, \alpha^{2} x_{0}\right)<(L-3 \eta) l_{\Sigma}\left(\alpha^{2}\right),
$$

which contradicts the fact that $\alpha^{2}$ is $(L, 3 \eta)$-straight and concludes the proof of Lemma 3.11(i).

To prove (ii), assume that $\gamma=a \alpha \beta \alpha b$ is $(L, \eta)$-straight, $l_{\Sigma}(\gamma)=6$ and $\alpha \beta \alpha$ is reduced. Lemma 3.10 says that $\alpha \beta \alpha$ is $(L, 2 \eta)$-straight and $\alpha \beta$ is $\left(L, C^{\prime} \eta\right)$-straight where $C^{\prime}=2 l_{\Sigma}(\alpha \beta \alpha) / l_{\Sigma}(\alpha \beta)$. Since $\alpha \beta \alpha$ is $(L, 2 \eta)$-straight, by the triangle inequality we have

$$
(L-2 \eta) l_{\Sigma}(\alpha \beta \alpha) \leq 2 \rho\left(x_{0}, \alpha x_{0}\right)+L l_{\Sigma}(\beta)
$$

and therefore

$$
2 \rho\left(x_{0}, \alpha x_{0}\right) \geq(L-2 \eta) l_{\Sigma}(\alpha \beta \alpha)-L l_{\Sigma}(\beta)=2 L l_{\Sigma}(\alpha)-2 \eta l_{\Sigma}(\alpha \beta \alpha),
$$


hence

$$
\rho\left(x_{0}, \alpha x_{0}\right) \geq L l_{\Sigma}(\alpha)-\eta l_{\Sigma}(\alpha \beta \alpha),
$$

and since $l_{\Sigma}(\alpha) \leq 2$ and $l_{\Sigma}(\beta) \leq 2$, we deduce that

$$
\rho\left(x_{0}, \alpha x_{0}\right) \geq(L-4 \eta) l_{\Sigma}(\alpha),
$$

that is, $\alpha$ is $(L, 4 \eta)$-straight. We set $x_{1}=\alpha \beta x_{0}, x_{2}=\alpha \beta \alpha x_{0}$ and $x_{3}=(\alpha \beta)^{2} x_{0}=$ $\alpha \beta \alpha \beta x_{0}$. We get, since $\alpha \beta \alpha$ is $(L, 2 \eta)$-straight,

$$
\begin{aligned}
\rho\left(x_{0}, x_{1}\right)+\rho\left(x_{1}, x_{2}\right)-\rho\left(x_{0}, x_{2}\right) & =\rho\left(x_{0}, \alpha \beta x_{0}\right)+\rho\left(x_{0}, \alpha x_{0}\right)-\rho\left(x_{0}, \alpha \beta \alpha x_{0}\right) \\
& \leq L\left[l_{\Sigma}(\alpha \beta)+l_{\Sigma}(\alpha)\right]-(L-2 \eta) l_{\Sigma}(\alpha \beta \alpha) \leq 12 \eta .
\end{aligned}
$$

In the same way, since $\alpha \beta$ is $\left(L, C^{\prime} \eta\right)$-straight with $C^{\prime}=2 l_{\Sigma}(\alpha \beta \alpha) / l_{\Sigma}(\alpha \beta)$, we have

$$
\begin{aligned}
\rho\left(x_{1}, x_{2}\right)+\rho\left(x_{2}, x_{3}\right)-\rho\left(x_{1}, x_{3}\right) & =\rho\left(x_{0}, \alpha x_{0}\right)+\rho\left(x_{0}, \beta x_{0}\right)-\rho\left(x_{0}, \alpha \beta x_{0}\right) \\
& \leq L\left[l_{\Sigma}(\alpha)+l_{\Sigma}(\beta)\right]-\left(L-C^{\prime} \eta\right) l_{\Sigma}(\alpha \beta) \\
& \leq 2 \eta l_{\Sigma}(\alpha \beta \alpha) \leq 12 \eta .
\end{aligned}
$$

We can therefore apply Lemma 3.6 to get, using also the triangle inequality,

$$
\begin{aligned}
2 \rho\left(x_{0}, \alpha \beta x_{0}\right)-\rho\left(x_{0},(\alpha \beta)^{2} x_{0}\right) & \leq \rho\left(x_{0}, \alpha \beta x_{0}\right)+\rho\left(x_{0}, \alpha x_{0}\right)+\rho\left(x_{0}, \beta x_{0}\right)-\rho\left(x_{0},(\alpha \beta)^{2} x_{0}\right) \\
& =\rho\left(x_{0}, x_{1}\right)+\rho\left(x_{1}, x_{2}\right)+\rho\left(x_{2}, x_{3}\right)-\rho\left(x_{0}, x_{3}\right) \\
& \leq\left(1+\frac{\rho\left(x_{0}, \beta x_{0}\right)}{\rho\left(x_{0}, \alpha x_{0}\right)}\right)\left(12 \eta+\operatorname{Argcosh} e^{12 \eta}\right) \\
& \leq\left(1+\frac{L l_{\Sigma}(\beta)}{(L-4 \eta) l_{\Sigma}(\alpha)}\right) \cdot \frac{1}{4}\left(1-\frac{e^{\delta}}{\cosh (L / 2)}\right)^{2},
\end{aligned}
$$

the last inequality coming from (25) and the fact that $\alpha$ is $(L, 4 \eta)$-straight. From (24), the fact that $l_{\Sigma}(\beta) \leq 2$ and $l_{\Sigma}(\alpha) \geq 1$ we get

$$
\rho\left(x_{0},(\alpha \beta)^{2} x_{0}\right)>2 \rho\left(x_{0}, \alpha \beta x_{0}\right)-\left(1-\frac{e^{\delta}}{\cosh (L / 2)}\right)^{2} .
$$

On the other hand we have seen that $\alpha \beta$ is $\left(L, C^{\prime} \eta\right)$-straight with $C^{\prime}=2 l_{\Sigma}(\alpha \beta \alpha) / l_{\Sigma}(\alpha \beta)$, so that

$$
\rho\left(x_{0}, \alpha \beta x_{0}\right) \geq\left(L-C^{\prime} \eta\right) l_{\Sigma}(\alpha \beta) \geq 2 L-2 \eta l_{\Sigma}(\alpha \beta \alpha),
$$

and since $l_{\Sigma}(\alpha \beta \alpha) \leq 6$ the above inequality gives, with (24),

$$
\rho\left(x_{0}, \alpha \beta x_{0}\right) \geq L .
$$

By assumption, since $l_{\Sigma}(\alpha \beta) \leq 4$, the displacement of $\alpha \beta$ satisfies $l(\alpha \beta) \leq \delta$, and by (27) we can apply Lemma 3.5 to get

$$
\rho\left(x_{0},(\alpha \beta)^{2} x_{0}\right) \leq 2 \rho\left(x_{0}, \alpha \beta x_{0}\right)-\left(1-\frac{e^{\delta}}{\cosh (L / 2)}\right)^{2},
$$

which contradicts (26). This concludes the proof of Lemma 3.11 and Proposition 3.9. 


\section{Mapping the Cayley graph of $G$ into $X$}

Let $G$ be a finitely generated discrete group of isometries of a Cartan-Hadamard manifold $(X, g)$ of sectional curvature $-a^{2} \leq K_{g} \leq-1$. We consider a finite generating set $S$ of $G$ and the Cayley graph $\mathcal{G}_{S}$ of $G$ associated to $S$. We define a distance $d_{S}$ on $\mathcal{G}_{S}$ in the following way: each edge is isometric to the segment $[0,1] \subset \mathbb{R}$ and the distance $d_{S}\left(\gamma, \gamma^{\prime}\right)$ between two vertices $\gamma, \gamma^{\prime}$ of $\mathcal{G}_{S}$ is the word distance $d_{S}\left(\gamma, \gamma^{\prime}\right)=l_{S}\left(\gamma^{-1} \gamma^{\prime}\right)$. The group $G$ acts by isometries on $\left(\mathcal{G}_{S}, d_{S}\right)$ and on $(X, g)$. The goal of this section is to construct for each number $c$ large enough an equivariant map $f_{c}: \mathcal{G}_{S} \rightarrow X$ such that $f_{c}$ is Lipschitzian with Lipschitz constant at most $c$.

\subsection{Poincaré series, measures and convexity}

We first consider the Poincaré series

$$
P_{c}(s, x, y)=\sum_{\gamma \in G} e^{-c d_{S}(s, \gamma)} \cosh [\rho(x, \gamma y)]
$$

where $c \in \mathbb{R}_{+}, s \in \mathcal{G}_{S}$ and $x, y \in X$.

Lemma 4.1. For all $s \in \mathcal{G}_{S}, x, y, x_{0}, y_{0} \in X, c>0$ and $\gamma_{0} \in G$ we have

(i) $P_{c}\left(\gamma_{0} s, \gamma_{0} x, y\right)=P_{c}(s, x, y)$,

(ii) $P_{c}(s, x, y) \leq P_{c}\left(s, x_{0}, y_{0}\right) e^{\rho\left(x_{0}, x\right)+\rho\left(y_{0}, y\right)}$.

In particular the convergence of the series is independent of the choice of the points $x, y \in X$.

Proof. The equivariance property of the Poincare series is straightforward. On the other hand by the triangle inequality we have

$$
\begin{aligned}
P_{c}(s, x, y) & =\sum_{\gamma \in G} e^{-c d_{S}(s, \gamma)} \cosh [\rho(x, \gamma y)] \\
& \leq \sum_{\gamma \in G} e^{-c d_{S}(s, \gamma)} \cosh \left[\rho\left(x_{0}, \gamma y_{0}\right)+\rho\left(x_{0}, x\right)+\rho\left(y_{0}, y\right)\right]
\end{aligned}
$$

hence

$$
P_{c}(s, x, y) \leq P_{c}\left(s, x_{0}, y_{0}\right) e^{\rho\left(x_{0}, x\right)+\rho\left(y_{0}, y\right)} .
$$

The critical exponent of this series is defined as

$$
c_{0}:=\inf \left\{c>0 \mid P_{c}(s, x, y)<\infty\right\} .
$$

Let $x_{0}$ be the point of $X$ such that $L(S)=\max _{i} \rho\left(x_{0}, \sigma_{i} x_{0}\right)$. By the triangle inequality, for all $\gamma \in \Gamma$ we have $\rho\left(x_{0}, \gamma x_{0}\right) \leq L(S) l_{S}(\gamma)$, therefore

$$
P_{c}\left(e, x_{0}, x_{0}\right) \leq \sum_{\gamma \in \Gamma} e^{-(c-L(S)) l_{S}(\gamma)} .
$$


On the other hand, by the definition of $\operatorname{Ent}_{S}(\Gamma)$, we have $\sum_{\gamma \in \Gamma} e^{-t l_{S}(\gamma)}<\infty$ for all $t>\operatorname{Ent}_{S}(\Gamma)$, hence we have proved that

$$
c_{0} \leq \operatorname{Ent}_{S}(\Gamma)+L(S) .
$$

From now on we only consider $c \in \mathbb{R}_{+}$such that $P_{c}(s, x, y)<\infty$.

Let us choose a probability measure $\mu$ with smooth density and compact support on $X$. For each $s \in \mathcal{G}_{S}$ define a measure on $X$ by

$$
\mu_{s}^{c}=\sum_{\gamma \in G} e^{-c d_{S}(s, \gamma)} \gamma_{*} \mu
$$

and a function $\mathcal{B}^{c}: \mathcal{G}_{S} \times X \rightarrow \mathbb{R}$ by

$$
\mathcal{B}^{c}(s, x)=\int_{X} \cosh [\rho(x, z)] d \mu_{s}^{c}(z) .
$$

In the following Lemmas 4.2, 4.3 and Corollary 4.4 we show that $x \mapsto \mathcal{B}^{c}(s, x)$ is a strictly convex $C^{2}$ function such that

$$
\lim _{x \rightarrow \infty} \mathcal{B}^{c}(s, x)=+\infty
$$

Lemma 4.2. Let $c$ be such that $P_{c}(s, x, y)<\infty$. For all $s \in \mathcal{G}_{S}$ and $x \in X$, we have $\mathcal{B}^{c}(s, x)<\infty$. Moreover, the function $x \mapsto \mathcal{B}^{c}(s, x)$ is strictly convex and $\lim _{x \rightarrow \infty} \mathcal{B}^{c}(s, x)=+\infty$.

Proof. By the definition of $\mu_{s}^{c}$,

$$
\mathcal{B}^{c}(s, x)=\int_{X} \sum_{\gamma \in G} e^{-c d_{S}(s, \gamma)} \cosh [\rho(x, \gamma z)] d \mu(z)=\int_{X} P_{c}(s, x, z) d \mu(z),
$$

so $\mathcal{B}^{c}(s, x)<\infty$ by Lemma 4.1(ii) since the support of $\mu$ is compact. For any geodesic $c(t)$ and $z$ in $X, t \mapsto d(c(t), z)$ is a convex function since $(X, g)$ has negative sectional curvature, therefore $t \mapsto \cosh [\rho(c(t), z)]$ is strictly convex and so is $x \mapsto \mathcal{B}^{c}(s, x)=$ $\int_{X} \cosh [\rho(x, z)] d \mu_{s}^{c}(z)$. On the other hand we have

$$
\mathcal{B}^{c}(s, x)=\int_{X} \cosh [\rho(x, z)] d \mu_{s}^{c}(z) \geq \frac{1}{2} e^{\rho\left(x, x_{0}\right)} \int_{X} e^{-\rho\left(x_{0}, z\right)} d \mu_{s}^{c}(z),
$$

so $\mathcal{B}^{c}(s, x) \rightarrow+\infty$ whenever $x$ tends to infinity in $X$.

In the above lemma we proved that $x \mapsto \mathcal{B}^{c}(s, x)$ is a strictly convex function which tends to $+\infty$ when $x$ tends to infinity. We shall now prove that $x \mapsto \mathcal{B}^{c}(s, x)$ is $C^{2}$. We will also give estimates of its second derivative. 
Lemma 4.3. Let $c$ be such that $P_{c}(s, x, y)<\infty$. The function $x \mapsto \mathcal{B}^{c}(s, x)$ is $C^{2}$ and for any $s \in \mathcal{G}_{S}, x \in X$ and any tangent vectors $v, w \in T_{x} X$ we have

$$
d \mathcal{B}^{c}(s, x)(v)=\int_{X} d \rho(x, z)(v) \sinh [\rho(x, z)] d \mu_{S}^{c}(z)
$$

and

$\operatorname{DdB}^{c}(s, x)(v, w)$

$=\int_{X}(\sinh [\rho(x, z)] D d \rho(x, z)(v, w)+\cosh [\rho(x, z)] d \rho(x, z) \otimes d \rho(x, z)(v, w)) d \mu_{s}^{c}(z)$.

Proof. Let $v \in T_{x} X$ be a unit tangent vector at a point $x \in X$. For each $z \neq x$ in $X$, we have

$$
d(\cosh [\rho(x, z)])(v)=d \rho(x, z)(v) \sinh [\rho(x, z)],
$$

hence

$$
|d(\cosh [\rho(x, z)])(v)|=|d \rho(x, z)(v) \sinh [\rho(x, z)]| \leq \cosh [\rho(x, z)],
$$

therefore $\cosh [\rho(x, z)] \leq 2 \cosh \left[\rho\left(x_{1}, z\right)\right]$ for $x$ in a sufficiently small neighbourhood of an arbitrary point $x_{1}$. Since $z \mapsto 2 \cosh \left[\rho\left(x_{1}, z\right)\right]$ is $\mu_{s}^{c}$-integrable, we can differentiate $x \mapsto \mathcal{B}^{c}(s, x)$ applying the Lebesgue differentiation theorem and get the first part of the statement.

Let us now compute the second derivative. We shall prove the equality for the quadratic form and get the general case by polarisation. Let $v \in T_{x} X$ be a unit tangent vector at $x \in X$. Let $\alpha(t)$ be the geodesic such that $\alpha(0)=x$ and $\alpha^{\prime}(0)=v$. We write $\rho_{(z, \alpha(t))}$ instead of $\rho(z, \alpha(t))$. Set

$$
h(t, z)=\frac{1}{t}\left(d \rho_{(z, \alpha(t))}\left(\alpha^{\prime}(t)\right) \sinh \left[\rho_{(z, \alpha(t))}\right]-d \rho_{(z, \alpha(0))}\left(\alpha^{\prime}(0)\right) \sinh \left[\rho_{(z, \alpha(0))}\right]\right) .
$$

When $z \neq x$ we have

$$
h_{0}(z):=\lim _{t \rightarrow 0} h(t, z)=\sinh \left[\rho_{(x, z)}\right] D d \rho_{(x, z)}(v, v)+\cosh \left[\rho_{(x, z)}\right] d \rho_{(x, z)} \otimes d \rho_{(x, z)}(v, v) \text {. }
$$

The formula which gives $D d \mathcal{B}^{c}(s, x)(v, w)$ in Lemma 4.3 is equivalent to

$$
\operatorname{DdB}^{c}(s, x)(v, v)=\int_{X} h_{0}(z) d \mu_{s}^{c}(z)
$$

and will be a consequence of Lebesgue's theorem. We need to show the existence of a $\mu_{s}^{c}$-integrable function $H$ such that for any $z \neq x$ and $t$ small enough so that $z \notin \alpha([0, t])$ we have $h(t, z) \leq H(z)$. To do this, first notice that $h(t, z)$ is non-negative since $D d \rho$ is, due to the negativity of the curvature. For each $z \notin \alpha([0, t])$ we have

$$
\begin{aligned}
0 \leq h(t, z) \leq \sup _{s \in[0, t]}\left[\sinh \left[\rho_{(z, \alpha(s))}\right] D d \rho_{(z, \alpha(s))}+\cdots\right. \\
\left.\cdots+\cosh \left[\rho_{(z, \alpha(s))}\right] d \rho_{(z, \alpha(s))} \otimes d \rho_{(z, \alpha(s))}\right]\left(\alpha^{\prime}(s), \alpha^{\prime}(s)\right) .
\end{aligned}
$$


Since the curvature of $(X, g)$ satisfies $-a^{2} \leq K_{g} \leq-1$, Rauch's comparison theorem shows that for each $x, y \in X$,

$$
D d \rho_{(x, y)} \leq a \frac{\cosh \left[a \rho_{(x, y)}\right]}{\sinh \left[a \rho_{(x, y)}\right]}\left(g-d \rho_{(x, y)} \otimes d \rho_{(x, y)}\right),
$$

hence from the previous inequality we get

$$
\begin{array}{r}
h(t, z) \leq \sup _{s \in[0, t]}\left[a \sinh \left[\rho_{(z, \alpha(s))}\right] \frac{\cosh \left[a \rho_{(z, \alpha(s))}\right]}{\sinh \left[a \rho_{(z, \alpha(s))}\right.}\left(g-d \rho_{(z, \alpha(s))} \otimes d \rho_{(z, \alpha(s))}\right)+\cdots\right. \\
\left.\cdots+\cosh \left[\rho_{(z, \alpha(s))}\right] d \rho_{(z, \alpha(s))} \otimes d \rho_{\left(z, \alpha\left(t^{\prime}\right)\right)}\right]\left(\alpha^{\prime}(s), \alpha^{\prime}(s)\right) .
\end{array}
$$

But since $a \geq 1$ the concavity of tanh on $\mathbb{R}_{+}$gives

$$
\frac{a}{\tanh a \rho} \geq \frac{1}{\tanh \rho}
$$

therefore we get

$$
0 \leq h(t, z) \leq a \sup _{s \in[0, t]} \sinh \left[\rho_{(z, \alpha(s))}\right] \frac{\cosh \left[a \rho_{(z, \alpha(s))}\right]}{\sinh \left[a \rho_{(z, \alpha(s))}\right]} .
$$

Finally, since $\sinh \rho \leq(1 / a) \sinh a \rho$, by convexity of sinh, we find that $0 \leq h(t, z) \leq$ $H(z)$ from (35) for all $|t| \leq 1 / a$ and all $z \notin \alpha([0, t])$ where

$$
H(z)= \begin{cases}a \frac{\cosh 1}{\sinh 1} \sinh \left[\rho_{(z, \alpha(0))}+1\right], & \rho_{(z, \alpha(0))} \geq 2 / a, \\ \cosh \left[a \rho_{(z, \alpha(0))}+1\right], & \rho_{(z, \alpha(0))}<2 / a,\end{cases}
$$

is $\mu_{s}^{c}$-integrable by Lemma 4.2. This concludes the proof of Lemma 4.3.

Lemma 4.3 has the following corollary.

Corollary 4.4. Under the assumptions of Lemma 4.3 we have

$$
D d \mathcal{B}^{c} \geq \mathcal{B}^{c} g,
$$

in particular, $\mathcal{B}^{c}$ is strictly convex.

Proof. Since the sectional curvature of $(X, g)$ satisfies $K_{g} \leq-1$ Rauch's theorem shows that

$$
D d \rho \geq \frac{1}{\tanh \rho}(g-d \rho \otimes d \rho) .
$$

From this inequality and Lemma 4.3 we therefore get, for all $x \in X$ and any unit tangent vector $v \in T_{X} X$,

$$
\operatorname{DdB}^{c}(v, v) \geq\left(\int_{X} \cosh \left[\rho_{(z, x)}\right] d \mu_{s}^{c}(z)\right) g(v, v)=\mathcal{B}^{c}(x) g(v, v) .
$$




\subsection{Construction of Lipschitzian maps $f_{c}: \mathcal{G}_{S} \rightarrow X$.}

So far we have shown that for any $s \in \mathcal{G}_{S}$ the function $x \mapsto \mathcal{B}^{c}(s, x)$ is strictly convex and tends to $+\infty$ as $x$ tends to infinity. We can then define a map $f_{c}: \mathcal{G}_{S} \rightarrow X$ as follows. For $s \in \mathcal{G}_{S}$ we define $f_{c}(s)$ as the unique point $x \in X$ which achieves the unique minimum of the function $x \mapsto \mathcal{B}^{c}(s, x)$. The rest of this section is devoted to proving

Proposition 4.5. Let $c$ be such that $P_{c}(s, x, y)<\infty$. Let $f_{c}:\left(\mathcal{G}_{S}, d_{S}\right) \rightarrow(X, g)$ associate to $s \in \mathcal{G}_{S}$ the unique point $x \in X$ which achieves the minimum of the function $x \mapsto \mathcal{B}^{c}(s, x)$. Then $f_{c}$ is Lipschitzian with Lipschitz constant $c$.

The proof of Proposition 4.5 relies on the following two technical lemmas.

Lemma 4.6. Let $c$ be such that $P_{c}(s, x, y)<\infty$. For all $x \in X$ and all tangent vectors $v \in T_{x} X$ the function $\alpha: s \mapsto d \mathcal{B}^{c}(s, x)(v)$ is differentiable at each point $s \in \mathcal{G}_{S}$ distinct from a vertex or the middle point of an edge. Moreover, for such an s we have

$$
\alpha^{\prime}(s)=-c \int_{X} d \rho_{(x, z)}(v) \sinh [\rho(x, z)] \sum_{\gamma \in G} \frac{d}{d s}\left(d_{S}(s, \gamma)\right) e^{-c d_{S}(s, \gamma)} d\left(\gamma_{*} \mu\right)(z) .
$$

Proof of Lemma 4.6. Let $\left[g, g^{\prime}\right]$ be the edge containing $s$ and parametrize it by $[0,1]$. We first observe that for all $\gamma \in G$,

$$
d_{S}(s, \gamma)=\min \left[d_{S}(g, \gamma)+t, d_{S}\left(g^{\prime}, \gamma\right)+1-t\right]
$$

where $t \in[0,1]$ is the parameter corresponding to $s$. Therefore $s \mapsto d_{S}(s, \gamma)$ is differentiable at each $s \in] g, g^{\prime}$ [ distinct from the middle point of ] $g, g^{\prime}[$. On the other hand, by Lemma 4.3,

$$
d \mathcal{B}^{c}(s, x)(v)=\int_{X} d \rho(x, z)(v) \sinh [\rho(x, z)] d \mu_{s}^{c}(z),
$$

so that we can write

$$
\begin{aligned}
\frac{1}{h}(\alpha(s+ & h)-\alpha(s)) \\
& =\sum_{\gamma \in G} \int_{X} d \rho_{(x, \gamma z)}(v) \sinh [\rho(x, \gamma z)] \frac{1}{h}\left[e^{-c d_{S}(s+h, \gamma)}-e^{-c d_{S}(s, \gamma)}\right] d \mu(z),
\end{aligned}
$$

where we have identified points in the edge $\left[g, g^{\prime}\right]$ with their parameters. Observe that for $|h|$ small enough,

$$
\left|\frac{1}{h}\left[e^{-c d_{S}(s+h, \gamma)}-e^{-c d_{S}(s, \gamma)}\right]\right| \leq 2 c e^{-c d_{S}(s, \gamma)},
$$

and

$$
2 c \sum_{\gamma \in G} \int_{X}\left|d \rho_{(x, \gamma z)}(v)\right| \sinh [\rho(x, \gamma z)] e^{-c d_{S}(s, \gamma)} d \mu(z)<\infty
$$


thanks to Lemma 4.2. Hence if $s \in \mathcal{G}_{S}$ is distinct from a vertex or the middle point of an edge we get

$$
\begin{aligned}
\lim _{h \rightarrow 0} \frac{1}{h}(\alpha & (s+h)-\alpha(s)) \\
& =-c \int_{X} d \rho_{(x, z)}(v) \sinh [\rho(x, z)] \sum_{\gamma \in G} \frac{d}{d s}\left(d_{S}(s, \gamma)\right) e^{-c d_{S}(s, \gamma)} d\left(\gamma_{*} \mu\right)(z)
\end{aligned}
$$

by Lebesgue's theorem.

Lemma 4.7. Let $c$ be such that $P_{c}(s, x, y)<\infty$. Let $s_{0} \in \mathcal{G}_{S}$ be a point distinct from a vertex or the middle point of an edge, and $u$ a unit vector tangent at $s_{0}$ to the edge containing $s_{0}$. Then $\left\|d f_{c}(u)\right\| \leq c$.

Proof. Fix a smooth moving frame $\left\{E_{1}, \ldots, E_{n}\right\}$ of $T X$ and define $\Phi: X \times \mathcal{G}_{S} \rightarrow \mathbb{R}^{n}$ by

$$
\Phi(x, s)=\left(d \mathcal{B}^{c}(s, x)\left(E_{1}\right), \ldots, d \mathcal{B}^{c}(s, x)\left(E_{n}\right)\right) .
$$

By definition, the point $f_{c}(s)$ is characterized by the implicit equation

$$
\Phi\left(f_{c}(s), s\right)=0,
$$

or equivalently,

$$
d \mathcal{B}^{c}\left(s, f_{c}(s)\right)=0 .
$$

For all $x \in X$ and $s \in \mathcal{G}_{S}$ in a neighbourhood of $s_{0}$ the function $\Phi$ is differentiable by Lemmas 4.3 and 4.6. Moreover since $x=f_{c}(s)$ is a critical point of the function $x \mapsto \mathcal{B}^{c}(s, x)$, we have, for $j=1, \ldots, n$,

$$
\frac{\partial \Phi}{\partial x}\left(f_{c}(s), s\right)\left(E_{j}\right)=\left(\operatorname{DdB}^{c}\left(s, f_{c}(s)\right)\left(E_{j}, E_{1}\right), \ldots, \operatorname{DdB}^{c}\left(s, f_{c}(s)\right)\left(E_{j}, E_{n}\right)\right),
$$

thus $\frac{\partial \Phi}{\partial x}\left(f_{c}(s), s\right)$ is invertible by Corollary 4.4. By the implicit function theorem, $f_{c}$ is differentiable in a neighbourhood of $s_{0}$, and if $u$ is a unit vector tangent at $s_{0}$ to the edge containing $s_{0}$, and $v$ a tangent vector in $T_{f_{c}(s)} X$, from the implicit equation we get

$$
\operatorname{DdB}^{c}\left(s_{0}, f_{c}\left(s_{0}\right)\right)\left(d f_{c}(u), v\right)=-\left.\frac{d}{d s}\right|_{s=s_{0}} d \mathcal{B}^{c}\left(s, f_{c}\left(s_{0}\right)\right)(v) .
$$

From Corollary 4.4 and Lemma 4.6 we obtain, setting $v=d f_{c}(u) /\left\|d f_{c}(u)\right\|$,

$$
\begin{aligned}
& \left|g\left(d f_{c}(u), v\right) \mathcal{B}^{c}\left(s_{0}, f_{c}\left(s_{0}\right)\right)\right| \\
& \leq c \int_{X}\left|d \rho_{\left(f_{c}\left(s_{0}\right), z\right)}(v)\right| \sinh \left[\rho\left(f_{c}\left(s_{0}\right), z\right)\right] \sum_{\gamma \in G}\left|\frac{d}{d s}\right|_{s=s_{0}}\left(d_{S}(s, \gamma)\right) \mid e^{-c d_{S}\left(s_{0}, \gamma\right)} d\left(\gamma_{*} \mu\right)(z),
\end{aligned}
$$

therefore

$$
\left|g\left(d f_{c}(u), v\right) \mathcal{B}^{c}\left(s_{0}, f_{c}\left(s_{0}\right)\right)\right| \leq c \int_{X} \sinh \left[\rho\left(f_{c}\left(s_{0}\right), z\right)\right] d \mu_{s_{0}}^{c}(z),
$$


hence

$$
\left\|d f_{c}(u)\right\| \leq c \frac{\int_{X} \sinh \left[\rho\left(f_{c}\left(s_{0}\right), z\right)\right] d \mu_{s_{0}}^{c}(z)}{\int_{X} \cosh \left[\rho\left(f_{c}\left(s_{0}\right), z\right)\right] d \mu_{s_{0}}^{c}(z)} \leq c .
$$

Proposition 4.5 then follows from

Corollary 4.8. Let c be such that $P_{c}(s, x, y)<\infty$. Then $f_{c}$ is Lipschitzian with Lipschitz constant $c$.

Proof. Let $\left[s_{1}, s_{2}\right] \subset \mathcal{G}_{S}$ be a segment which contains no vertices or middle points. It directly follows from Lemma 4.7 that

$$
\rho\left(f_{c}\left(s_{1}\right), f_{c}\left(s_{2}\right)\right) \leq c d_{S}\left(s_{1}, s_{2}\right) .
$$

We now want to extend the inequality (38) to all points $s_{1}, s_{2} \in \mathcal{G}_{S}$. For that purpose we first consider a segment $\left[s_{1}, s_{2}\right] \subset \mathcal{G}_{S}$ where $s_{1}$ is the midpoint of an edge, and $s_{2}$ a vertex of the same edge; the inequality (38) for these points $s_{1}, s_{2}$ follows from the continuity of $f_{c}$ at $s_{1}$ and $s_{2}$ proved below. Corollary 4.8 will then follow from the fact that any segment $\left[s_{1}, s_{2}\right] \subset \mathcal{G}_{S}$ can be decomposed into a finite sequence of adjacent intervals $\left[y_{1}^{k}, y_{2}^{k}\right]$ where $y_{1}^{k}$ is the midpoint and $y_{2}^{k}$ a vertex of the same edge or the other way around, except for the first and last intervals.

Let us now prove the continuity of $f_{c}$ at a vertex or the midpoint $s$ of an edge. Given such a point $s$, let $\left\{s_{k}\right\}_{k \in \mathbb{N}}$ be a sequence converging to $s$ and staying in a single mid-edge containing $s$. The sequence $x_{k}:=f_{c}\left(s_{k}\right)$ is a Cauchy sequence in $X$ by (38) whose limit is a point $x=\lim _{k} x_{k}$. We want to prove that $f_{c}(s)=x$. For all $z \in X$ and $k \in \mathbb{N}$ we have

$$
\mathcal{B}^{c}\left(s_{k}, z\right) \geq \mathcal{B}^{c}\left(s_{k}, x_{k}\right)
$$

by the definition of $x_{k}=f_{c}\left(s_{k}\right)$. We claim that $\lim _{k} \mathcal{B}^{c}\left(s_{k}, x_{k}\right)=\mathcal{B}^{c}(s, x)$ and $\lim _{k} \mathcal{B}^{c}\left(s_{k}, z\right)=\mathcal{B}^{c}(s, z)$. Assuming the claim and taking the limit in (39) as $k \rightarrow \infty$ gives, for all $z \in X$,

$$
\mathcal{B}^{c}(s, z) \geq \mathcal{B}^{c}(s, x)
$$

therefore $x=f_{c}(s)$.

We now prove the claim. By (30) and (31), we have

$$
\mathcal{B}^{c}\left(s_{k}, x_{k}\right)=\int_{X} \cosh \left[\rho\left(x_{k}, z\right)\right] d \mu_{s_{k}}^{c}(z)=\int_{X} \sum_{\gamma \in G} e^{-c d_{S}\left(s_{k}, \gamma\right)} \cosh \left[\rho\left(x_{k}, \gamma z\right)\right] d \mu(z)
$$

Since $e^{-c d_{S}\left(s_{k}, \gamma\right)} \cosh \left[\rho\left(x_{k}, \gamma z\right)\right] \leq e^{c} e^{-c d_{S}(s, \gamma)} \cosh [\rho(x, \gamma z)+1]$ for $k$ large enough, we get $\lim _{k} \mathcal{B}^{c}\left(s_{k}, x_{k}\right)=\mathcal{B}^{c}(s, x)$ by Lebesgue's theorem. Similarly $\lim _{k} \mathcal{B}^{c}\left(s_{k}, z\right)=$ $\mathcal{B}^{c}(s, z)$, which concludes the proof of the claim, Corollary 4.8 and Proposition 4.5. 


\section{Algebraic entropy and $\eta$-straight isometries}

Let $G$ be a finitely generated discrete group of isometries of $(X, g)$ whose sectional curvature satisfies $-a^{2} \leq K_{g} \leq-1$, and $S=\left\{\sigma_{1}, \ldots, \sigma_{p}\right\}$ be a finite generating set.

We assume that the minimal displacement $L(S)=\inf _{x \in X} \max _{i=1, \ldots, p} \rho\left(x, \sigma_{i} x\right)$ of $S$ (cf. Definition 2.2) satisfies $L(S)>0$. By Lemma 3.3 there exists a point $x_{0} \in X$ such that

$$
L(S)=\inf _{x \in X} \max _{i \in\{1, \ldots, p\}} \rho\left(x, \sigma_{i} x\right)=\max _{i \in\{1, \ldots, p\}} \rho\left(x_{0}, \sigma_{i} x_{0}\right) .
$$

The goal of this section is to prove that if all elements of $G$ are "almost non- $\eta$-straight" for some $\eta$ such that $L(S)>\eta>0$, then the entropy of $G$ with respect to $S$ is bounded below by $\eta$. By an "almost non- $\eta$-straight" isometry $\gamma$ we mean that $\rho\left(x_{0}, \gamma x_{0}\right) \leq(L(S)-$ $\eta) l_{S}(\gamma)+D$ for some positive $D$.

Theorem 5.1. Let $G$ be a finitely generated discrete group of isometries of $(X, g)$ whose sectional curvature satisfies $-a^{2} \leq K_{g} \leq-1$, and $S=\left\{\sigma_{1}, \ldots, \sigma_{p}\right\}$ be a finite generating set of $G$ with $L(S)>0$. Assume that there exist $D \geq 0$ and $\eta, 0<\eta<L(S)$, such that for all $\gamma \in G$,

$$
\rho\left(x_{0}, \gamma x_{0}\right) \leq(L(S)-\eta) l_{S}(\gamma)+D .
$$

Then $\operatorname{Ent}_{S}(G) \geq \eta$.

Proof. The proof relies on the construction made in Section 4 of an equivariant Lipschitzian map with Lipschitz constant $c>\operatorname{Ent}_{S}(G)+L(S)-\eta$.

Let us prove that under the assumption (41) for any $c>\operatorname{Ent}_{S}(G)+L(S)-\eta$ we have $P_{c}(s, x, y)<\infty$. By the triangle inequality,

$$
e^{-c d_{S}(s, \gamma)} \leq e^{c d_{S}(s, e)} e^{-c d_{S}(\gamma, e)},
$$

and for any $x_{0} \in X$,

$$
\cosh [\rho(x, \gamma y)] \leq e^{\rho(x, \gamma y)} \leq e^{\rho\left(x, x_{0}\right)+\rho\left(x_{0}, \gamma x_{0}\right)+\rho\left(x_{0}, y\right)} .
$$

Therefore, for $x_{0}, D$ and $\eta$ such that (41) holds, we get

$$
P_{c}(s, x, y) \leq e^{D+c d_{S}(e, s)+\rho\left(x, x_{0}\right)+\rho\left(x_{0}, y\right)} \sum_{\gamma \in G} e^{[L(S)-\eta-c] d_{S}(e, \gamma)},
$$

and so $P_{c}(s, x, y)<\infty$ for each $c>\operatorname{Ent}_{S}(G)+L(S)-\eta$.

Hence by Proposition 4.5 there exists an equivariant Lipschitzian map $f_{c}:\left(\mathcal{G}_{S}, d_{S}\right)$ $\rightarrow(X, g)$ with Lipschitz constant $c$, for any $c>\operatorname{Ent}_{S}(G)+L(S)-\eta$. We consider the point $f_{c}(e)$, where $e$ is the neutral element of $G$. By the definition of $L(S)$, there is a $\sigma_{i} \in S$ such that $\rho\left(f_{c}(e), \sigma_{i}\left(f_{c}(e)\right)\right) \geq L(S)$. Therefore, by equivariance,

$$
\rho\left(f_{c}(e), \sigma_{i}\left(f_{c}(e)\right)\right)=\rho\left(f_{c}(e), f_{c}\left(\sigma_{i}(e)\right)\right) \geq L(S) .
$$

On the other hand, since $f_{c}$ is $c$-Lipschitzian we have

$$
\rho\left(f_{c}(e), f_{c}\left(\sigma_{i}(e)\right)\right) \leq c d_{S}\left(e, \sigma_{i}(e)\right)=c .
$$

The above two inequalities give

$$
c \geq L(S)
$$

and since $c$ is any number such that $c>\operatorname{Ent}_{S}(G)+L(S)-\eta$, we get $\operatorname{Ent}_{S}(G) \geq \eta$. 


\section{Proof of the main theorem}

In this section we shall first prove that the entropy of a group with respect to a set of two generators with displacement $L>0$ is bounded below. Then we shall prove the main theorem.

Proposition 6.1. Let $(X, g)$ be a Cartan-Hadamard manifold whose sectional curvature satisfies $-a^{2} \leq K_{g} \leq-1$, and $G$ a non-virtually nilpotent discrete group of isometries of $(X, g)$ generated by two isometries $\left\{\sigma_{1}, \sigma_{2}\right\}$. Assume that

$$
L=\inf _{x \in X} \max \left\{\rho\left(x, \sigma_{1} x\right), \rho\left(x, \sigma_{2} x\right)\right\}>0 .
$$

Then the entropy of $G$ relative to the set of generators $\Sigma=\left\{\sigma_{1}, \sigma_{2}\right\}$ satisfies

$$
\operatorname{Ent}_{\Sigma}(G) \geq \min \left[\frac{\log \cosh (L / 4)}{5+\log \cosh (L / 4)} \frac{\log 2}{6}, \frac{1}{1000}\left(1-\frac{\cosh (L / 4)}{\cosh (L / 2)}\right)^{4}\right]
$$

Proof. Let $\delta=\log \cosh (L / 4)$. The proof is divided into two cases. In the first case we can find two elements in $G$ of bounded length $l_{\Sigma}$ which are hyperbolic with distinct axes and displacement larger than $\delta$. In that case, a classical ping-pong argument shows that the semigroup generated by these two elements (or their inverses) is free with corresponding entropy bounded below by a constant depending on $\delta$. In the second case, when we cannot find such a free semigroup, we can show that all elements of $G$ are almost non- $\eta$-straight for some $\eta=\eta(\delta, L)$ and we conclude using Theorem 5.1. More precisely the two cases are:

Case 1. There exists an element $\gamma \in G$ of algebraic length $l_{\Sigma}(\gamma) \leq 4$ whose displacement $l(\gamma)$ in $X$ satisfies $l(\gamma)>\delta$.

Case 2. The displacement of all elements $\gamma \in G$ of algebraic length $l_{\Sigma}(\gamma) \leq 4$ satisfies $l(\gamma) \leq \delta$.

In Case 1 , let $\gamma \in G$ be of algebraic length $l_{\Sigma}(\gamma) \leq 4$ and with $l(\gamma)>\delta$. We note that $\gamma$ is then a hyperbolic isometry of $X$. Since $G$ is not virtually nilpotent, one of the generators $\sigma_{1}$ or $\sigma_{2}$, say $\sigma_{1}$, does not preserve the axis of $\gamma$. Indeed if both $\sigma_{1}$ and $\sigma_{2}$ preserved the axis of $\gamma$, then so would $G$, and hence it would be virtually abelian by Lemma 2.5(ii), a contradiction. Thus, if $(\theta, \eta)$ are the endpoints of the axis of $\gamma$, then $\sigma_{1}(\{\theta, \eta\}) \cap\{\theta, \eta\}=\varnothing$ by the proof of Lemma 2.5(i). We can now apply the effective ping-pong lemma proved in the appendix to the two hyperbolic elements $\gamma$ and $\sigma_{1} \gamma \sigma_{1}^{-1}$ which have disjoint fixed-point sets. This shows that the algebraic entropy of the subgroup generated by $\gamma$ and $\sigma_{1} \gamma \sigma_{1}^{-1}$ is bounded below by $\frac{\delta}{5+\delta} \log 2$. We then deduce that

$$
\operatorname{Ent}_{\Sigma}(\Gamma) \geq \frac{\delta}{5+\delta} \frac{\log 2}{6} .
$$

In Case 2, Proposition 3.9 tells us that all elements $\gamma \in G$ of length $l_{\Sigma}(\gamma)=6$ are non- $(L, \eta)$-straight where $\eta$ is given by $(23), \eta=10^{-3}\left(1-\frac{\cosh (L / 4)}{\cosh (L / 2)}\right)^{4}$. Thus, every element $g \in \Gamma$ of algebraic length 6 satisfies

$$
\rho\left(x_{0}, g x_{0}\right) \leq(L-\eta) l_{\Sigma}(g)
$$


Hence, every element $\gamma \in \Gamma$ satisfies

$$
\rho\left(x_{0}, \gamma x_{0}\right) \leq(L-\eta)\left(l_{\Sigma}(\gamma)-5\right)+5 L .
$$

Therefore Theorem 5.1 yields $\operatorname{Ent}_{\Sigma}(G) \geq \eta=10^{-3}\left(1-\frac{\cosh (L / 4)}{\cosh (L / 2)}\right)^{4}$.

We can now prove the main theorem which we recall below.

Theorem 6.2 (Main theorem). Let $(X, g)$ be a Cartan-Hadamard manifold whose sectional curvature satisfies $-a^{2} \leq K_{g} \leq-1$. Let $\Gamma$ be a discrete and finitely generated subgroup of the isometry group of $(X, g)$. Then either $\Gamma$ is virtually nilpotent or its algebraic entropy is bounded below by an explicit constant $C(n, a)$.

Remark 6.3. The constant is

$C(n, a)=\min \left[\frac{\log \cosh (\mu(n, a) / 4)}{5+\log \cosh (\mu(n, a) / 4)} \frac{\log 2}{12}, \frac{1}{2000}\left(1-\frac{\cosh (\mu(n, a) / 4)}{\cosh (\mu(n, a) / 2)}\right)^{4}\right.$,

$\left.\mu(n, a) / 4, \frac{1}{4}\left(1-\frac{1}{\cosh \mu(n, a)}\right)^{2}, \frac{1}{2} \log \left(\frac{1}{2}\left(\cosh (\mu(n, a) / 2)+\frac{1}{\cosh (\mu(n, a) / 2)}\right)\right)\right]$.

Proof. If $S=\left\{\sigma_{1}, \ldots, \sigma_{p}\right\}$ is a finite generating set of $\Gamma$, Proposition 2.6 allows us to reduce the proof to the following three cases:

(i) There exist $\sigma_{i}, \sigma_{j} \in S$ such that $L\left(\left\langle\sigma_{i}, \sigma_{j}\right\rangle\right) \geq \mu(n, a)$ and $\left\langle\sigma_{i}, \sigma_{j}\right\rangle$ is not virtually nilpotent.

(ii) There exist $\sigma_{i}, \sigma_{j}, \sigma_{k} \in S$ such that $L\left(\left\langle\sigma_{i} \sigma_{j}, \sigma_{k}\right\rangle\right) \geq \mu(n, a)$ and $\left\langle\sigma_{i} \sigma_{j}, \sigma_{k}\right\rangle$ is not virtually nilpotent.

(iii) All $\sigma_{i}$ 's are elliptic and, for all $i \neq j$, the subgroup $\left\langle\sigma_{i}, \sigma_{j}\right\rangle$ fixes a point $y \in X$ or a point $\theta \in \partial X$.

In the first (resp. second) case Proposition 6.1 gives a lower bound for the algebraic entropy of $\left\langle\sigma_{i}, \sigma_{j}\right\rangle$ (resp. $\left\langle\sigma_{i} \sigma_{j}, \sigma_{k}\right\rangle$ ) with respect to the generating set $\left\{\sigma_{i}, \sigma_{j}\right\}$ (resp. $\left.\left\{\sigma_{i} \sigma_{j}, \sigma_{k}\right\}\right)$ by the number

$$
\min \left[\frac{\log \cosh (\mu(n, a) / 4)}{5+\log \cosh (\mu(n, a) / 4)} \frac{\log 2}{6}, \frac{1}{1000}\left(1-\frac{\cosh (\mu(n, a) / 4)}{\cosh (\mu(n, a) / 2)}\right)^{4}\right],
$$

using the fact that $L\left(\sigma_{i}, \sigma_{j}\right) \geq \mu(n, a)$ (resp. $L\left(\sigma_{i} \sigma_{j}, \sigma_{k}\right) \geq \mu(n, a)$ ). We conclude in cases (i) and (ii) by noticing that the entropy of $\Gamma$ with respect to $S$ is bounded below by $\operatorname{Ent}_{\left\{\sigma_{i}, \sigma_{j}\right\}}\left(\left\langle\sigma_{i}, \sigma_{j}\right\rangle\right)$ (resp. by $\left.\frac{1}{2} \operatorname{Ent}_{\left\{\sigma_{i} \sigma_{j}, \sigma_{k}\right\}}\left(\left\langle\sigma_{i} \sigma_{j}, \sigma_{k}\right\rangle\right)\right)$, since $d_{\left\{\sigma_{i}, \sigma_{j}\right\}} \geq d_{S}$ (resp. $\left.d_{\left\{\sigma_{i} \sigma_{j}, \sigma_{k}\right\}} \geq \frac{1}{2} d_{S}\right)$.

In the third case, Proposition 3.8 implies that

$$
\rho\left(x_{0}, \gamma x_{0}\right) \leq(L(S)-\eta / 2)\left(l_{S}(\gamma)-1\right)+L(S),
$$

where $\eta$ is given in Proposition 3.8. We conclude by applying Theorem 5.1, which gives $\operatorname{Ent}_{S}(\Gamma) \geq \eta$, and then bounding below $\eta$ using $L(S) \geq \mu(n, a)$. 


\section{Appendix}

In this section $(X, g)$ is a Cartan-Hadamard manifold of sectional curvature $K_{g} \leq-1$. It is well known that if $\alpha, \beta$ are two hyperbolic isometries of $(X, g)$ with disjoint axes, then for $N$ sufficiently large, $\alpha^{N}$ and $\beta^{N}$ generate a non-abelian free subgroup of Isom $X$. In [Gro81], [De196], it was shown that if $\Gamma$ is a hyperbolic group then $N$ can be chosen independent of $\alpha$ and $\beta$ in $\Gamma$ and under the same assumptions the number $N$ was shown to depend only on the number of generators and the constant of hyperbolicity of $\Gamma$ [Ch-G00]. In what follows we show that $N=N(\delta)$ can be chosen depending only on $\delta>0$ which bounds from below the displacement of two hyperbolic isometries of $(X, g), \alpha$ and $\beta$, with disjoint fixed-point sets.

Proposition 7.1. Let $(X, g)$ be a Cartan-Hadamard manifold of sectional curvature $K_{g} \leq-1$, and $\Gamma$ a discrete subgroup of $\operatorname{Isom}(X, g)$. Assume that $\alpha$ and $\beta$ have disjoint fixed-point sets and their displacements satisfy $l(\alpha) \geq \delta$ and $l(\beta) \geq \delta$, where $\delta$ is a positive number. Then $\left(\alpha^{N}, \beta^{N}\right)$ or $\left(\alpha^{N}, \beta^{-N}\right)$ generates a non-abelian free semigroup, where $N=E(5 / \delta)+1$ and $E(x)$ stands for the integer part of $x$.

Before proceeding to the proof of Proposition 7.1 let us set some notation. Denote by $x=$ $x(t)$ and $y=y(t), t \in \mathbb{R}$, the axes of $\alpha$ and $\beta$. The points $\theta^{ \pm}=\lim _{t \rightarrow \pm \infty} x(t)$ and $\zeta^{ \pm}=$ $\lim _{t \rightarrow \pm \infty} y(t)$ are the fixed points of $\alpha$ and $\beta$ on the ideal boundary $\partial X$ of $X$. Denote by $x^{+}$and $x^{-}$the projections of $\zeta^{+}$and $\zeta^{-}$on the axis of $\alpha$. We can assume that $x^{+}$is closer to $\theta^{+}$than $x^{-}$(if not, we replace $\beta$ by $\beta^{-1}$ ). Also denote by $y_{0}$ the projection of $x^{+}$on the axis of $\beta$. We now parametrize $x$ and $y$ in such a way that $x(0)=x^{+}$and $y(0)=y_{0}$. We set $t_{1}=N l(\alpha)=l\left(\alpha^{N}\right)$ and $t_{2}=N l(\beta)=l\left(\beta^{N}\right)$, where $N=E(5 / \delta)+1$. We define $U^{ \pm}$as the set of points $p \in X$ such that $\rho\left(p, x\left( \pm t_{1}\right)\right) \leq \rho(p, x(0))$. In the same way we define $V^{ \pm}$as the set of points $p \in X$ such that $\rho\left(p, y\left( \pm t_{2}\right)\right) \leq \rho(p, y(0))$. For a unit tangent vector $u \in T_{x} X$ at a point $x \in X$ and $\alpha \in[0, \pi$ [ we define $\mathcal{C}(u, \alpha)=$ $\left\{\exp _{x} v: v \in T_{x} X, \angle(u, v) \in\left[0, \alpha[\}\right.\right.$, the cone of angle $\alpha$ around $u$ at $x$, where $\exp _{x}$ is the exponential map at $x$.

We further need the following geometric lemmas. For a triangle $A B C$ in $(X, g)$, we will write $\hat{A}$ for the angle at $A$, and $a, b, c$ for the lengths of the sides opposite to $A, B, C$.

Lemma 7.2. Let $A B C$ be a triangle in $(X, g)$.

(i) If $\pi / 6 \leq \hat{A} \leq \pi$, then $\rho(B, C)>\rho(A, B)+\rho(A, C)-4$.

(ii) If $\hat{A} \geq \pi / 2 \leq \pi$, then $\rho(B, C)>\rho(A, B)+\rho(A, C)-1$.

Proof. Since $K_{g} \leq-1$, we have

$$
\cosh a \geq \cosh b \cosh c-\cos \hat{A} \sinh b \sinh c .
$$

The first inequality of Lemma 7.2 will therefore be a consequence of the fact that if $b+c>4$ then

$$
\cosh (b+c-4)-\cosh b \cosh c+\cos \hat{A} \sinh b \sinh c<0 .
$$


Setting $X=e^{-(b+c)}$ we have

$\cosh (b+c-4)-\cosh b \cosh c+\cos \hat{A} \sinh b \sinh c$

$$
=\frac{1}{4} e^{(b+c)}\left[\left(2 e^{4}-1+\cos \hat{A}\right) X^{2}-\left(e^{-2 b}+e^{-2 c}\right)(1+\cos \hat{A})-\left(1-\cos \hat{A}-2 e^{-4}\right)\right] .
$$

Since $e^{-2 b}+e^{-2 c} \geq 2 e^{-(b+c)}$, we then get

$$
\cosh (b+c-4)-\cosh b \cosh c+\cos \hat{A} \sinh b \sinh c \leq e^{(b+c)} P(X)
$$

where

$$
P(X)=\left(2 e^{4}-1+\cos \hat{A}\right) X^{2}-2(1+\cos \hat{A}) X-\left(1-\cos \hat{A}-2 e^{-4}\right)
$$

and $P(X)$ is negative when $P(0)<0$ and $P\left(e^{-4}\right)<0$, which is the case if $\cos \hat{A}<$ $1-2 e^{-4}$ and so when $\hat{A} \geq \pi / 6$. This proves the first inequality of the lemma. The second inequality is proved similarly when $\cos \hat{A}<1-2 e^{-1}$.

Lemma 7.3. The sets $U^{+}$and $U^{-}$are contained in $\mathcal{C}(\dot{x}(0), \pi / 6)$ and $\mathcal{C}(-\dot{x}(0), \pi / 6)$ respectively.

Proof. We recall that $x(0)=x^{+}$. Let $c(t)$ be a geodesic ray starting at $x^{+}$such that $\angle(\dot{x}(0), \dot{c}(0)) \geq \pi / 6$. Since $t_{1} \geq 5$, Lemma 7.2 implies

$$
\rho\left(c(t), x\left(t_{1}\right)\right)>\rho\left(x^{+}, c(t)\right)+\rho\left(x^{+}, x\left(t_{1}\right)\right)-4 \geq \rho\left(c(t), x^{+}\right),
$$

therefore $c(t) \notin U^{+}$. The same argument holds for $U^{-}$.

Let $z_{t}$ be the geodesic joining $x^{+}$and $y(t)$, and $z_{ \pm \infty}$ the geodesic joining $x^{+}$and $y( \pm \infty)$ $=\zeta^{ \pm}$.

Lemma 7.4. The set $V^{ \pm}$is contained in $\mathcal{C}\left(\dot{z}_{ \pm \infty}(0), \pi / 3\right)$.

Proof. Recall that the angle at $y(0)=y_{0}$ between $z_{0}$ and $y$ is equal to $\pi / 2$, so that Lemma 7.2 says that

$$
\text { length }\left(z_{t}\right)>\text { length }\left(z_{0}\right)+t-1 \text {, }
$$

and in particular,

$$
\text { length }\left(z_{t_{2}}\right)>\text { length }\left(z_{0}\right)+t_{2}-1 \text {. }
$$

Let us now show that $\angle\left(\dot{z}_{t_{2}}(0), \dot{z}_{+\infty}(0)\right) \leq \pi / 6$. Assume for contradiction that $\angle\left(\dot{z}_{t_{2}}(0), \dot{z}_{+\infty}(0)\right)>\pi / 6$. Then by Lemma 7.2 we have, as $t \rightarrow \infty$,

$$
t-t_{2}>\text { length }\left(z_{t_{2}}\right)+\text { length }\left(z_{t}\right)-4
$$

but summing up (44) and (45) leads to a contradiction with (46) since $t_{2} \geq 5$. Therefore $\angle\left(\dot{z}_{t_{2}}(0), \dot{z}_{+\infty}(0)\right) \leq \pi / 6$. Now consider a geodesic ray $c$ starting at $x^{+}$such that $\angle\left(\dot{c}(0), \dot{z}_{+\infty}(0)\right) \geq \pi / 3$. Thus, $\angle\left(\dot{c}(0), \dot{z}_{t_{2}}(0)\right) \geq \pi / 6$ and by Lemma 7.2 we get

$$
\rho\left(c(t), y\left(t_{2}\right)\right)>\rho\left(c(t), x^{+}\right)+\operatorname{length}\left(z_{t_{2}}\right)-4,
$$


and applying again (45),

$$
\rho\left(c(t), y\left(t_{2}\right)\right)>\rho\left(c(t), x^{+}\right)+\text {length }\left(z_{0}\right)+t_{2}-5 .
$$

The last inequality becomes, by the triangle inequality,

$$
\rho\left(c(t), y\left(t_{2}\right)\right)>\rho\left(c(t), y_{0}\right)+t_{2}-5,
$$

therefore $\rho\left(c(t), y\left(t_{2}\right)\right)>\rho\left(c(t), y_{0}\right)$ since $t_{2} \geq 5$.

We have proved that a geodesic ray $c$ starting at $x^{+}$such that $\angle\left(\dot{c}(0), \dot{z}_{+\infty}(0)\right) \geq \pi / 3$ does not intersect $V^{+}$. This proves that $V^{+} \subset \mathcal{C}\left(\dot{z}_{+\infty}(0), \pi / 3\right)$. By the same argument we also have $V^{-} \subset \mathcal{C}\left(\dot{z}_{-\infty}(0), \pi / 3\right)$, which ends the proof of the lemma.

Lemma 7.5. We have $U^{+} \cap U^{-}=U^{+} \cap V^{+}=U^{+} \cap V^{-}=U^{-} \cap V^{+}=V^{+} \cap V^{-}=\emptyset$.

Proof. Since $\angle\left(\dot{x}(0), \dot{z}_{+\infty}(0)\right)=\pi / 2, \angle\left(\dot{x}(0), \dot{z}_{-\infty}(0)\right) \geq \pi / 2, \angle(\dot{x}(0),-\dot{x}(0))=\pi$, and from the relative position of $x^{+}, x^{-}$and $\theta^{+}$, it follows that $\mathcal{C}(\dot{x}(0), \pi / 6)$ does not intersect $\mathcal{C}\left(\dot{z}_{+\infty}(0), \pi / 3\right), \mathcal{C}(-\dot{x}(0), \pi / 6)$ or $\mathcal{C}\left(\dot{z}_{-\infty}(0), \pi / 3\right)$. Therefore by Lemmas 7.3, 7.4 we conclude that $U^{+}$does not intersect $U^{-}, V^{+}$or $V^{-}$. Now since $\angle\left(-\dot{x}(0), \dot{z}_{+\infty}(0)\right)$ $=\pi / 2$, we have $\mathcal{C}\left(\dot{z}_{+\infty}(0), \pi / 3\right) \cap \mathcal{C}(-\dot{x}(0), \pi / 6)=\emptyset$, hence $V^{+} \cap U^{-}=\emptyset$. If $p \in$ $V^{+} \cap V^{-}$, we have $\rho(p, y(0)) \geq \rho\left(p, y\left(-t_{2}\right)\right)$ and $\rho(p, y(0)) \geq \rho\left(p, y\left(t_{2}\right)\right)$, which contradicts the convexity of the function $t \mapsto \rho(y(t), p)$. Therefore $V^{+} \cap V^{-}=\emptyset$.

Lemma 7.6. We have $\alpha^{N}\left(V^{+}\right) \subset U^{+}$and $\beta^{N}\left(U^{+}\right) \subset V^{+}$.

Proof. Since $x$ and $y$ are the axes of $\alpha^{N}$ and $\beta^{N}$ respectively we have $\alpha^{N}\left(x\left(-t_{1}\right)\right)=$ $x(0), \beta^{N}\left(y\left(-t_{1}\right)\right)=y(0), \alpha^{N}(x(0))=x\left(t_{1}\right)$ and $\beta^{N}(y(0))=y\left(t_{2}\right)$. Therefore for any $p \in X-U^{-}$we have $\alpha^{N}(p) \in U^{+}$, and similarly for any $p \in X-V^{-}$we have $\beta^{N}(p) \in V^{+}$, by the definition of $N$. On the other hand, by Lemma 7.5, $V^{+} \subset X-U^{-}$ and $U^{+} \subset X-V^{-}$, which concludes the proof.

The proof of Proposition 7.1 is a direct application of Lemma 7.6 by a standard ping-pong argument.

Acknowledgments. The authors wish to thank Luc Guyot for valuable comments on an earlier version of this text. This work has been partially supported by the ANR-FOG, grant ANR-07-BLAN0251 .

\section{References}

[AN05] Alperin, R. C., Noskov, G. A.: Nonvanishing of algebraic entropy for geometrically finite groups of isometries of Hadamard manifolds. Int. J. Algebra Comput. 15, 799813 (2005) Zbl 1107.20026 MR 2197807

[Bal195] Ballmann, W.: Lectures on Spaces of Nonpositive Curvature. DMV Seminar 25, Birkhäuser (1995) Zbl 0834.53003 MR 1377265

[BCG] Besson, G., Courtois, G., Gallot, S.: A Margulis lemma without curvature. In preparation.

[Bre08] Breuillard, E.: A strong Tits alternative. arXiv:0804.1395 (2008) 
[BrGe08] Breuillard, E., Gelander, T.: Uniform dependence in linear groups. Invent. Math. 173, 225-263 (2008) Zbl 1148.20029 MR 2415307

[Bur-Zal] Burago, Y., Zalgaller, V. A.: Geometric Inequalities. Grundlehren Math. Wiss. 285, Springer, Berlin (1988) Zbl 0633.53002 MR 0936419

[Ch-G00] Champetier, C., Guirardel, V.: Monoïdes libres dans les groupes hyperboliques. In: Séminaires de théorie spectrale et géométrie 18, Univ. de Grenoble I, 157-170 (2000) Zbl 0973.20036 MR 1812218

[Har02] de la Harpe, P.: Uniform growth in groups of exponential growth. Geom. Dedicata 95, 1-17 (2002) Zbl 1025.20027 MR 1950882

[De196] Delzant, Th.: Sous-groupes distingués et quotients des groupes hyperboliques. Duke Math. J. 83, 661-682 (1996) Zbl 0852.20032 MR 1390660

[Ebe96] Eberlein, P. B.: Geometry of Non-Positively Curved Manifolds. Chicago Lectures in Math., Univ of Chicago Press, Chicago, IL (1996) Zbl 0883.53003 MR 1441541

[EMO05] Eskin, A., Mozes, S., Oh, H.: On uniform exponential growth for linear groups. Invent. Math. 160, 1-30 (2005) Zbl 1137.20024 MR 2129706

[Gro87] Gromov, M.: Hyperbolic groups. In: Essays in Group Theory, Math. Sci. Res. Inst. Publ. 8, Springer, New York, 75-263 (1987) Zbl 0634.20015 MR 0919829

[Gro81] Gromov, M., Lafontaine, J., Pansu, P.: Structures métriques pour les variétés riemanniennes. Cedic/Nathan (1981) Zbl 0509.53034 MR 0682063

[Kou98] Koubi, M.: Croissance uniforme dans les groupes hyperboliques. Ann. Inst. Fourier (Grenoble) 48, 1441-1453 (1998) Zbl 0914.20033 MR 1662255

[Osi03] Osin, D. V.: The entropy of solvable groups. Ergodic Theory Dynam. Systems 23, $907-$ 918 (2003) Zbl 1062.20039 MR 1992670

[Wil04] Wilson, J. S.: On exponential growth and uniformly exponential growth for groups. Invent. Math. 155, 287-303 (2004) Zbl 1065.20054 MR 2031429

[Wol68] Wolf, J. A.: Growth of finitely generated solvable groups and curvature of Riemannian manifolds. J. Differential Geom. 2, 421-446 (1968) Zbl 0207.51803 MR 0248688 